\title{
Review Article \\ Consecutive-Type Reliability Systems: An Overview and Some Applications
}

\author{
Ioannis S. Triantafyllou \\ Department of Statistics and Insurance Science, University of Piraeus, 80 Karaoli and Dimitriou Street, 18534 Piraeus, Greece \\ Correspondence should be addressed to Ioannis S. Triantafyllou; itrantal@unipi.gr
}

Received 28 July 2014; Revised 10 April 2015; Accepted 17 April 2015

Academic Editor: Xiaohu Li

Copyright (C) 2015 Ioannis S. Triantafyllou. This is an open access article distributed under the Creative Commons Attribution License, which permits unrestricted use, distribution, and reproduction in any medium, provided the original work is properly cited.

The family of consecutive-type reliability systems is under investigation. More specifically, an up-to-date presentation of almost all generalizations of the well-known consecutive $k$-out-of- $n: F$ system that have been proposed in the literature is displayed, while several recent and fundamental results for each member of the aforementioned family are stated.

\section{Introduction}

A linear (circular) consecutive $k$-out-of- $n: F$ system consists of $n$ components which are linearly (circularly) arranged and the system fails if and only if at least $k$ consecutive components fail. The most popular applications of these systems pertain to computer network, telecommunication, pipeline network modeling, engineering, or integrated circuitry design. The consecutive $k$-out-of- $n$ : $F$ system has been subject of substantial research interest for many years and a lot of generalizations have been suggested in order to accommodate more flexible operation principles. For a detailed presentation of the consecutive $k$-out-of- $n$ : $F$ systems and some generalizations, the interested reader is referred to the excellent monograph of Kuo and Zuo [1] or the work of Chang et al. [2].

Let $T$ be the lifetime of a reliability system with $n$ components and $X_{1}, X_{2}, \ldots, X_{n}$ its components' lifetimes. If we assume that $X_{1}, X_{2}, \ldots, X_{n}$ are exchangeable (and therefore identically distributed but not necessarily independent), the signature of the system is defined as the probability vector $\left(s_{1}(n), s_{2}(n), \ldots, s_{n}(n)\right)$ with

$$
s_{i}(n)=P\left(T=X_{i: n}\right), \quad i=1,2, \ldots, n,
$$

where $X_{1: n} \leq X_{2: n} \leq \cdots \leq X_{n: n}$ are the order statistics of the sample $X_{1}, X_{2}, \ldots, X_{n}$. It can be easily verified that, in the exchangeable case, the signature of a reliability system depends only on its structure and not on the specific underlying distribution of $X_{i}$. In other words, $s_{i}(n)$ is the proportion of permutations, among the $n$ ! equally likely permutations of $X_{1}, X_{2}, \ldots, X_{n}$, which result in a minimal cut set failing upon the occurrence of $X_{i: n}$.

The signature of the system, which was first introduced by Samaniego [3], is closely related to many well-known reliability characteristics, a fact turning it to a very useful tool for studying coherent systems and their ageing properties. For example, the reliability polynomial of a structure can be easily expressed in terms of its signature. More precisely, Samaniego [3] proved that for any coherent system with independent and identical components which have absolutely continuous cumulative density functions, system's reliability can be expressed as

$$
R(t)=P(T>t)=\sum_{i=1}^{n} s_{i}(n) P\left(X_{i: n}>t\right) .
$$

Navarro and Rychlik [4] proved that the above representation also holds true when the lifetimes $X_{1}, X_{2}, \ldots, X_{n}$ have an absolutely continuous exchangeable distribution (this property had been mentioned earlier by Kochar et al. [5]). Moreover, Eryilmaz and Bayramoglu [6] used the system signature in order to evaluate the extreme residual lifetimes of the remaining components after the complete failure of the system.

Both reliability function and signature of a structure can be evaluated based on either recursive formulas or 
explicit expressions. In some cases, where neither of the aforementioned methods can be established for a specific system, approximating and limiting results are available. In the present review article, the family of consecutive-type reliability systems is under investigation. More specifically, Section 2 offers an up-to-date presentation of almost all generalizations of the well-known consecutive $k$-out-of- $n$ : $F$ system that have been proposed and studied in the literature. For each structure, the corresponding fundamental work and selected results are displayed either briefly or in detail. In Section 3, applications of these reliability systems in several fields are described, while Section 4 presents a full-detailed diagram which connects the aforementioned structures by giving the information under which conditions a system can be treated as a special case of another one.

\section{Family of Consecutive-Type Reliability Systems}

In this section, we study in detail almost all generalizations of the well-known consecutive $k$-out-of- $n$ : $F$ systems that have been proposed in the literature till now. For each reliability structure that is included in the family of consecutivetype systems, the general operational structure is described, while several important and some recent relevant results are displayed.

2.1. $m$-Consecutive-k-out-of- $n$ : F Systems. An $m$-consecutive$k$-out-of- $n$ : $F$ system consists of $n$ components and fails if and only if there exist at least $m$ nonoverlapping runs of $k$ consecutive failed components. This system was first introduced by Griffith [7] and since then it has attracted a considerable research attraction. In the sequel, we present the main results appearing in the literature, such as recurrence relations and closed formulas for the evaluation of the reliability function and signature vector of $m$-consecutive- $k$-out-of- $n$ : $F$ systems. It is worth mentioning that the aforementioned system generalizes the well-known consecutive- $k$-out-of- $n$ : $F$ system (for $m=1$ ), while for $k=1$ the $m$-consecutive- $k$-out-of- $n$ : $F$ system reduces to an ordinary $m$-out-of- $n: F$ system.

2.1.1. Recursive Schemes for the Reliability of $m$-Consecutive- $k$ out-of-n: F Systems. The following theorems provide recurrence for the calculation of reliability of an $m$-consecutive- $k$ out-of- $n$ : $F$ system.

Theorem 1 (Papastavridis [8]). Let $R_{m, k, n}^{L}\left(p_{1}, p_{2}, \ldots, p_{n}\right)$ denote the reliability function of a linear $m$-consecutive- $k$-outof- $n$ : $F$ system, where $p_{i}$ is the reliability of its ith component. Then $R_{m, k, n}^{L}\left(p_{1}, p_{2}, \ldots, p_{n}\right)$ satisfies the following recurrence relation:

$$
\begin{aligned}
& R_{m, k, n}^{L}\left(p_{1}, p_{2}, \ldots, p_{n}\right)=R_{m, k, n}^{L}\left(p_{1}, p_{2}, \ldots, p_{n}\right) \\
& \quad-\sum_{s=1}^{m} p_{n-s k} \prod_{i=1}^{s k} q_{n-s k+i}\left[R_{m-s+1, k, n-s k-1}^{L}\left(p_{1}, p_{2}, \ldots, p_{n}\right)\right. \\
& \left.\quad-R_{m-s, k, n-s k-1}^{L}\left(p_{1}, p_{2}, \ldots, p_{n}\right)\right]
\end{aligned}
$$

where $n \geq k m+1$ and $R_{0, k, 0}^{L}\left(p_{1}, p_{2}, \ldots, p_{n}\right)=0$.
In order to launch the above recurrence scheme, a set of initial of conditions would be necessary. Observing that for $n \geq k+1$ the following ensues

$$
\begin{aligned}
& R_{1, k, n}^{L}\left(p_{1}, p_{2}, \ldots, p_{n}\right)=R_{1, k, n-1}^{L}\left(p_{1}, p_{2}, \ldots, p_{n}\right) \\
& \quad-p_{n-k}\left(1-p_{n-k+1}\right) \cdots\left(1-p_{n}\right) \\
& \quad \cdot R_{1, k, n-k-1}^{L}\left(p_{1}, p_{2}, \ldots, p_{n}\right)
\end{aligned}
$$

while

$$
R_{m, k, m k}^{L}\left(p_{1}, p_{2}, \ldots, p_{n}\right)=1-\prod_{i=1}^{n}\left(1-p_{i}\right)
$$

we have at hand the set of initial values needed to evaluate reliability of the system.

Note that for the i.i.d case (e.g., $p_{1}=p_{2}=\cdots=p_{n}=p$ ), the recurrence of Theorem 1 reduces to the following form:

$$
\begin{aligned}
& R_{m, k, n}^{L}(p) \\
& =R_{m, k, n-1}^{L}(p) \\
& \quad-\sum_{s=1}^{m} p q^{s k}\left[R_{m-s+1, k, n-s k-1}^{L}(p)-R_{m-s, k, n-s k-1}^{L}(p)\right], \\
& n \geq k m+1 .
\end{aligned}
$$

Theorem 2 (Alevizos et al. [9]). Let $R_{m, k, n}^{C}\left(p_{1}, p_{2}, \ldots, p_{n}\right)$ denote the reliability function of a circular $m$-consecutive- $k$ out-of- $n$ : $F$ system, where $p_{i}\left(q_{i}=1-p_{i}\right)$ is the reliability (unreliability) of its ith component. Then $R_{m, k, n}^{C}\left(p_{1}, p_{2}, \ldots, p_{n}\right)$ satisfies the following recurrence:

$$
\begin{aligned}
& R_{m, k, n}^{C}\left(p_{1}, p_{2}, \ldots, p_{n}\right)=p_{n} R_{m, k, n-1}^{L}\left(p_{1}, p_{2}, \ldots, p_{n}\right) \\
& \quad-p_{n}-q_{n} R_{m, k, n-1}^{C}\left(p_{1}, p_{2}, \ldots, p_{n}\right) \\
& \quad-\sum_{s=1}^{m} \sum_{i=0}^{s k-1}\left(q_{1} q_{2} \cdots q_{i} p_{i+1}\right) \\
& \cdot\left(q_{n} q_{n-1} \cdots q_{n-s k+i+1} p_{n-s k+i}\right) \\
& \quad \times\left(R_{m-s+1, k, n-s k-2}^{L, i+2}\left(p_{1}, p_{2}, \ldots, p_{n}\right)\right. \\
& \left.-R_{m-s, k, n-s k-2}^{L, i+2}\left(p_{1}, p_{2}, \ldots, p_{n}\right)\right)
\end{aligned}
$$

where $n \geq k m+2$ and $R_{d, k, c}^{L, i+2}\left(p_{1}, p_{2}, \ldots, p_{n}\right)$ denotes the reliability of a linear $d$-consecutive- $k$-out-of-c: $F$ subsystem with components $i+2, i+3, \ldots, n-s k+i-1$.

To launch the above recursive scheme, a set of initial of conditions would be necessary. Observing that the following ensue

(i) $R_{m, k, n}^{C}=1$, for $n<k m$,

(ii) $R_{m, k, n}^{C}=1-\prod_{i=1}^{n} q_{i}$, for $n=k m$,

(iii) $R_{m, k, n}^{C}=1-\prod_{i=1}^{n} q_{i}-\sum_{i=1}^{n} q_{1} q_{2} \cdots q_{i-1} p_{i} q_{i+1} \cdots q_{n}$, for $n=k m+1$, 
we have at hand the set of initial values needed to evaluate reliability of the system.

Note that for the i.i.d case (e.g., $p_{1}=p_{2}=\cdots=p_{n}=p$ ), the recurrence of Theorem 2 reduces to the following form:

$$
\begin{gathered}
R_{m, k, n}^{C}(p)=p R_{m, k, n-1}^{L}(p)-p-q R_{m, k, n-1}^{C}(p) \\
-\sum_{s=1}^{m} \sum_{i=0}^{s k-1} p^{2} q^{s k}\left(R_{m-s+1, k, n-s k-2}^{L}(p)\right. \\
\left.-R_{m-s, k, n-s k-2}^{L}(p)\right), \quad n \geq k m+2 .
\end{gathered}
$$

It is worth mentioning that the computational complexity of recurrence included in Theorem 1 is equal to $O(n m)$, while the corresponding one for equations of Theorem 2 equals $\mathrm{O}\left(\mathrm{nkm}^{3}\right)$.

2.1.2. Exact Formulas for the Reliability of $m$-Consecutive$k$-out-of- $n$ : F Systems. The following theorems offer closed expressions for the evaluation of reliability of an $m$ consecutive- $k$-out-of- $n$ : $F$ system.

Theorem 3 (Papastavridis [8]). Let $R_{m, k, n}^{L}(p)$ denote the reliability function of a linear $m$-consecutive- $k$-out-of- $n$ : $F$ system, where $p$ is the common reliability of its i.i.d. components. Then $R_{m, k, n}^{L}(p)$ is given as follows:

$$
\begin{aligned}
R_{m, k, n}^{L}(p)= & \sum_{s=m}^{s^{*}} \sum_{i=s k}^{n}\left(\begin{array}{c}
s+n-i \\
s
\end{array}\right) N(i-s k, n-i+1) \\
& \cdot(1-p)^{i} p^{n-i},
\end{aligned}
$$

where $n \geq k m, s^{*}(x)$ denotes the greatest integer lower bound of $x$ and $N(i, j)$ can be expressed as

$$
N(i, j)=\sum_{s=0}^{j}(-1)^{s}\left(\begin{array}{l}
j \\
s
\end{array}\right)\left(\begin{array}{c}
i+j-1-s k \\
i-s k
\end{array}\right) .
$$

Theorem 4 (Makri and Philippou [10]). Let $R_{m, k, n}^{L}(p)$ denote the reliability function of a linear $m$-consecutive-k-out-of-n: $F$ system composed by i.i.d. components, where $p$ is their common reliability. Then $R_{m, k, n}^{L}(p)$ is given as follows:

$$
\begin{aligned}
& R_{m, k, n}^{L}(p) \\
& \quad=\sum_{x=0}^{m-1} \sum_{i=0}^{k-1} \sum_{x_{1}, x_{2}, \ldots, x_{k}}\left(\begin{array}{c}
x_{1}+x_{2}+\cdots+x_{k}+x \\
x_{1}, x_{2}, \ldots, x_{k}, x
\end{array}\right) \\
& \cdot(1-p)^{n}\left(\frac{p}{1-p}\right)^{x_{1}+x_{2}+\cdots+x_{k}},
\end{aligned}
$$

where

$$
\left(\begin{array}{c}
a \\
b_{1}, b_{2}, \ldots, b_{c}
\end{array}\right)=\frac{a !}{b_{1} ! b_{2} ! \cdots b_{c} !}
$$

is the well-known multinomial coefficient, while the inner summation is over all nonnegative integers $x_{1}, x_{2}, \ldots, x_{k}$ such that $\sum_{j=1}^{k} j x_{j}=n-i-k x$.
Theorem 5 (Makri and Philippou [10]). Let $R_{m, k, n}^{C}(p)$ denote the reliability function of a circular $m$-consecutive- $k$-out-of- $n$ : F system, where $p(q)$ is the common reliability (unreliability) of its components. Then, $R_{m, k, n}^{C}(p)$ can be expressed as follows:

(i)

$$
R_{m, k, n}^{C}(p)=\sum_{x=0}^{m-1} M_{x, c}(q ; k, n)
$$

where

$$
\begin{aligned}
& M_{x, c}(q ; k, n)=p(1-p)^{n-1} \\
& \cdot \sum_{i=1}^{k} i \sum\left(\begin{array}{c}
x_{1}+x_{2}+\cdots+x_{k}+x \\
x_{1}, x_{2}, \ldots, x_{k}, x
\end{array}\right) \\
& \cdot\left(\frac{p}{1-p}\right)^{x_{1}+x_{2}+\cdots+x_{k}} \\
& +k p q^{n-1} \sum_{i=1}^{k} \sum \frac{x}{x_{1}+x_{2}+\cdots+x_{k}+1} \\
& \quad \times\left(\begin{array}{c}
x_{1}+x_{2}+\cdots+x_{k}+x \\
x_{1}, x_{2}, \ldots, x_{k}, x
\end{array}\right)\left(\frac{p}{q}\right)^{x_{1}+x_{2}+\cdots+x_{k}} \\
& +q^{n} \delta_{x,[n / k]}
\end{aligned}
$$

is the well-known multinomial coefficient, while the inner summation is overall nonnegative integers $x_{1}, x_{2}, \ldots, x_{k}$ such that $\sum_{j=1}^{k} j x_{j}=n-i-k x$. Moreover, in the above expression $[x]$ denotes the greatest integer in $x$, while $\delta_{i, j}$ is the Kronecker delta function.

(ii) Consider

$$
R_{m, k, n}^{C}(p)=\sum_{x=0}^{m-1} B_{x, c}(q ; k, n)
$$

where

$$
\begin{aligned}
& B_{x, c}(q ; k, n)=q^{n} \delta_{x,[n / k]} \\
& +\sum_{y=[(n-k x-1) / k]}^{n-k x-1} q^{n-y-1} p^{y+1} \sum_{j=0}^{[(n-k x-1-y) / k]}(-1)^{j} \\
& +\left[\left(\begin{array}{c}
y+x \\
y
\end{array}\right)\left(\begin{array}{c}
y+1 \\
j
\end{array}\right)\left(\begin{array}{c}
n-k x-j k \\
y+1
\end{array}\right)\right. \\
& +k\left(\begin{array}{c}
y+x \\
y+1
\end{array}\right)\left(\begin{array}{c}
y \\
j
\end{array}\right)\left(\begin{array}{c}
n-k x-j k-1 \\
y
\end{array}\right) \\
& \left.-k\left(\begin{array}{c}
y+x+1 \\
y+1
\end{array}\right)\left(\begin{array}{c}
y \\
j
\end{array}\right)\left(\begin{array}{c}
n-k x-1-(j+1) k \\
y
\end{array}\right)\right] .
\end{aligned}
$$


2.1.3. Approximations for the Reliability of $m$-Consecutive$k$-out-of-n: F Systems. The following theorems offer some limiting results for the evaluation of reliability of an $m$ consecutive- $k$-out-of- $n$ : $F$ system.

Theorem 6 (Papastavridis [8]). Let $q(t)=(\lambda t)^{a}+O\left(t^{a}\right)$ be the common failure distribution of the components of an $m$-consecutive- $k$-out-of- $n$ : $F$ system, where $\lambda$, a are positive constants. Then the following ensues

$$
\lim _{n \rightarrow \infty} P\left(n^{1 / k a} T_{n}>t\right)=\exp \left[-(\lambda t)^{a k}\right] \sum_{i=0}^{m-1} \frac{(\lambda t)^{a k i}}{i !}
$$

where $T_{n}$ is the time of first failure of the system.

Theorem 7 (Godbole [11]). Let $q_{j}=1-p_{j}, j=1,2, \ldots, n$ be the failure probability of the jth component of an $\mathrm{m}$ consecutive- $k$-out-of- $n$ : F system. Then the reliability $R_{m, k, n}\left(p_{j}\right)$ of the system satisfies the following inequality:

$$
\left|R_{m, k, n}\left(p_{j}\right)-\sum_{x=0}^{m-1} \exp \frac{\left(-\lambda_{n}\right) \lambda_{n}^{x}}{x !}\right| \leq\left(2 k+2+n q^{k}\right) q^{k}
$$

where $q=\max _{j \geq 1} q_{j}$ and $\lambda_{n}=\sum_{j=k+1}^{n} p_{j-k} q_{j-k+1} \cdots q_{j}$.

2.1.4. Signature Vector of $m$-Consecutive-k-out-of- $n$ : F Systems. Let $p$ denote the common reliability of the components of an $m$-consecutive- $k$-out-of- $n$ : $F$ system. The following theorem offers a generating function approach of the aforementioned system.

Theorem 8. Let $\left(s_{1}(n, k, m), s_{2}(n, k, m), \ldots, s_{n}(n, k, m)\right)$ and $R_{m, k, n}(p)$ be the signature and the reliability function of an $m$ consecutive-k-out-of- $n$ : $F$ system, respectively. Then

(a) the double generating function of $i\left(\begin{array}{c}n \\ i\end{array}\right) s_{i}(n, k, m)$ is given by

$$
\begin{aligned}
\sum_{n=1}^{\infty} \sum_{i=1}^{n} i\left(\begin{array}{l}
n \\
i
\end{array}\right) s_{i}(n, k, m) t^{i} x^{n} \\
=\frac{m(t x)^{k m}(t x-1)^{m-1}\left\{k-k t x+t x\left[(t x)^{k}-1\right]\right\}}{\left\{x\left[1+t-(t x)^{k}\right]-1\right\}^{m+1}}
\end{aligned}
$$

(Eryilmaz et al. [12]),

(b) the generating function of $R_{m, k, n}(p)$ is given as follows:

$$
\begin{aligned}
r(z) & =\sum_{n=0}^{\infty} R_{n, m, k}(p) z^{n} \\
& =\frac{1}{1-z}-\frac{(q z)^{m k}}{(1-z)\left(1-p z \sum_{j=0}^{k-1}(q z)^{j}\right)^{m}}
\end{aligned}
$$

(Koutras [13]).

The next theorem offers expressions for the evaluation of the signature vector of an $m$-consecutive- $k$-out-of- $n$ : F system.
Theorem 9 (Eryilmaz et al. [12]). Let $\left(s_{1}(n, k, m), s_{2}(n, k, m)\right.$, $\left.\ldots, s_{n}(n, k, m)\right)$ be the signature vector of an $m$-consecutive- $k$ out-of-n: $F$ system. Then the following ensues

(a) the quantities $s_{i}(n, k, m)$ satisfy the recurrence relation:

$$
\begin{aligned}
& i\left(\begin{array}{c}
n \\
i
\end{array}\right) s_{i}(n, k, m) \\
& \quad=\sum_{l=1}^{k}(i-l+1)\left(\begin{array}{c}
n-l \\
i-l+1
\end{array}\right) s_{i-l+1}(n-l, k, m) \\
& \quad+\frac{m}{m-1}(i-k)\left(\begin{array}{c}
n-k \\
i-k
\end{array}\right) s_{i-k}(n-k, k, m-1)
\end{aligned}
$$

$$
\text { for } i=1,2, \ldots, n \text { and } n \geq k+1 \text {. }
$$

(b) the quantities $s_{i}(n, k, m)$ can be expressed as

$$
\begin{gathered}
s_{i}(n, k, m)=\left(\begin{array}{c}
n \\
i
\end{array}\right)^{-1}\left[\sum_{s=m}^{[i / k]}\left(\begin{array}{c}
s+n-i \\
s
\end{array}\right)\right. \\
\cdot C(i-s k, k, n-i+1)-\frac{n-i+1}{i} \\
\cdot \sum_{s=m}^{[(i-1) / k]}\left(\begin{array}{c}
s+n-i+1 \\
s
\end{array}\right) \\
\cdot C(i-s k-1, k, n-i+2)]
\end{gathered}
$$

for $m k \leq i \leq n$ and $s_{i}(n, k, m)=0$ if $i<m k$, where

$$
C(i, k, j)=\sum_{s=0}^{j}(-1)^{s}\left(\begin{array}{l}
j \\
s
\end{array}\right)\left(\begin{array}{c}
i+j-1-s k \\
i-s k
\end{array}\right) .
$$

2.1.5. Additional Results for $m$-Consecutive- $k$-out-of- $n$ : F Systems. Beyond the results mentioned in the previous subsections, additional studies have appeared in the literature for the $m$-consecutive- $k$-out-of- $n$ : $F$ system. Eryilmaz [14] derived explicit expressions for the component importance measures for the aforementioned structure consisting of exchangeable components. More specifically, Eryilmaz [14] studied in detail the well-known Birnbaum and BarlowProschan importance measures for a $m$-consecutive- $k$-outof- $n$ : $F$ system. Furthermore, Ghoraf [15] offered recursive formulas for calculating the reliability function of the circular case of the aforementioned structure couching on the corresponding recurrence equations for the linear one.

2.2. $r$-within-Consecutive-k-out-of-n: $F$ Systems. An $r$ within-consecutive- $k$-out-of- $n: F$ system consists of $n$ components and fails if and only if there exist $k$ consecutive components which include among them at least $r$ failed components. This system was first introduced by Griffith [7], but its mathematical modelling has been done earlier by Greenberg [16] and Saperstein [17]. In the sequel, we 
present the main results appearing in the literature, such as recurrence relations and closed formulas for the evaluation of the reliability function and signature vector of $r$-withinconsecutive- $k$-out-of- $n$ : $F$ systems. It is worth mentioning that the aforementioned system generalizes the well-known consecutive- $k$-out-of- $n$ : $F$ system (for $r<k$ ), while for $k=n$ the $r$-within-consecutive- $k$-out-of- $n$ : $F$ system reduces to an ordinary $r$-out-of- $n$ : $F$ system.

2.2.1. Recursive Schemes for the Reliability of $r$-within-Consecutive-k-out-of-n: F Systems. The following theorems provide recurrence for the calculation of reliability of an $r$-withinconsecutive- $k$-out-of- $n$ : $F$ system.

Theorem 10 (Sfakianakis et al. [18]). Let $R_{r, k, n}^{L}(p)$ denote the reliability function of a linear $r$-within-consecutive- $k$-out-of- $n$ : $F$ system, where $p$ is the common reliability of its components. Then, for $n=r+\lambda, \lambda \leq r, R_{r, k, n}^{L}(p)$ satisfies the following recurrence relation:

$$
\begin{aligned}
& R_{r, k, n}^{L}(p) \\
& \quad=\sum_{x=1}^{k} R_{x, \lambda, 2 \lambda}^{L}(p)\left(\begin{array}{c}
k-\lambda \\
r-x
\end{array}\right) p^{k-\lambda-r+x}(1-p)^{r-x},
\end{aligned}
$$

where $R_{x, \lambda, 2 \lambda}^{L}(p)=1$, if $x>\lambda$.

Theorem 11 (Eryilmaz [19]). Let $R_{r, k, n}^{L}(p)$ denote the reliability function of a linear $r$-within-consecutive- $k$-out-of- $n$ : $F$ system, where $p$ is the common reliability of its components. Then, for $n \leq 2 k, R_{r, k, n}^{L}(p)$ satisfies the following recurrence relation:

$$
\begin{array}{r}
R_{r, k, n}^{L}(t)=\sum_{s=0}^{\min (n-k, r-1)} P\left(T_{r-s: 2 k-n}^{[n-k+1: k]}>t\right) \\
\cdot\left[R_{s+1, n-k: 2(n-k)}^{*}(t)-R_{s, n-k: 2(n-k)}^{*}(t)\right],
\end{array}
$$

where $R_{s, n-k: 2(n-k)}^{*}(t)$ is the reliability of $s$-within-consecutive$(n-m)$-out-of-2(n-m): $F$ system with components $1,2, \ldots, n-$ $m, m+1, \ldots, n$, while $T_{k: m}^{[i: i+m-1]}$ denotes the lifetime of $k$ out-of-m: $F$ subsystem of components with the lifetimes $T_{i}, T_{i+1}, \ldots, T_{i+m-1}, 1 \leq i \leq n-m+1$.

Theorem 12 (Koutras [13]). Let $R_{r, k, n}^{L}(p)$ denote the reliability function of a linear $r$-within-consecutive- $k$-out-of- $n$ : $F$ system, where $p$ is the common reliability of its components. Then, $R_{r, k, n}^{L}(p)$ satisfies the following recurrence relation:

$$
\begin{aligned}
& R_{r, k, n}^{L}(p) \\
& = \begin{cases}1, & \text { for } n=0, \\
p R_{r, k, n-1}(p)+q p^{n-1}, & \text { for } 1 \leq n \leq k-1, \\
p R_{r, k, n-1}(p)+q p^{k-1} R_{r, k, n-k}(p), & \text { for } n \geq k .\end{cases}
\end{aligned}
$$

2.2.2. Exact Formulas for the Reliability of $r$-within-Consecutive-k-out-of-n: F Systems. The following Theorem offers closed expressions for the evaluation of reliability of an $r$ within-consecutive- $k$-out-of- $n$ : $F$ system.
Theorem 13 (Sfakianakis et al. [18]). Let $R_{2, k, n}^{L}(p)\left(R_{2, k, n}^{C}(p)\right)$ denote the reliability function of a linear (circular) 2-withinconsecutive-k-out-of-n: $F$ system, where $p$ is the common reliability of its components. Then the following recurrences ensue:

(i)

$$
R_{2, k, n}^{L}(p)=\sum_{j=0}^{m}\left(\begin{array}{c}
n-(j-1)(k-1) \\
j
\end{array}\right)(1-p)^{j} p^{n-j}
$$

where $m=[(n+k-1) / k]$,

$$
\begin{aligned}
& R_{2, k, n}^{C}(p) \\
& \quad=\sum_{j=0}^{s} \frac{n}{n-j(k-1)}\left(\begin{array}{c}
n-j(k-1) \\
j
\end{array}\right)(1-p)^{j} p^{n-j},
\end{aligned}
$$

where $m=[n / k]$.

2.2.3. Approximations for the Reliability of $r$-within-Consecutive-k-out-of- $n$ : $F$ Systems. The following theorems offer some limiting results for the evaluation of reliability of an $r$ within-consecutive- $k$-out-of- $n$ : $F$ system.

Theorem 14 (Eryilmaz et al. [20]). Let $R_{r, k, n}^{L}(t)=P\left(T_{r, k, n}>t\right)$ denote the reliability function of a linear $r$-within-consecutive$k$-out-of- $n$ : $F$ system. Then, for $1 \leq r \leq k \leq n, R_{r, k, n}^{L}(t)$ satisfies the following inequalities:

(i)

$$
\begin{aligned}
R_{r, k, n}^{L}(t) \geq & 1-(n-k+1) P\left(T_{r: k}^{(1)} \leq t\right) \\
& +(n-k) P\left(T_{r: k}^{(1)} \leq t, T_{r: k}^{(2)} \leq t\right),
\end{aligned}
$$

where $T_{r: k}^{(j)}$ is the rth smallest lifetime among $T_{j}, T_{j+1}$, $\ldots, T_{j+k-1}, r \leq k, 1 \leq j \leq n-k+1$ and

$$
\begin{aligned}
& P\left(T_{r: k}^{(1)} \leq t\right) \\
& \quad=\sum_{s=r}^{k}\left(\begin{array}{l}
k \\
s
\end{array}\right) \sum_{i=0}^{r-s}(-1)^{i}\left(\begin{array}{c}
r-s \\
i
\end{array}\right) P\left(T_{1} \leq t, \ldots, T_{s+i} \leq t\right) .
\end{aligned}
$$

(ii) Consider

$$
\begin{aligned}
& R_{r, k, n}^{L}(t) \\
& \quad \leq \sum_{j_{1}, j_{2}, \ldots, j_{h}=0}^{r-1}\left(\begin{array}{l}
k \\
j_{1}
\end{array}\right) \cdots\left(\begin{array}{l}
k \\
j_{h}
\end{array}\right) f\left(h k-\sum_{i=1}^{h} j_{i}, \sum_{i=1}^{h} j_{i}\right),
\end{aligned}
$$

where $h=[n / k]$ and

$$
f(a, b)=\sum_{i=0}^{a}(-1)^{i}\left(\begin{array}{l}
a \\
i
\end{array}\right) P\left(T_{1} \leq t, \ldots, T_{b+i} \leq t\right) .
$$


Let us next denote by $A_{i}$ the event where there are at least $r$ failed components from $i$ to $i+k-1$, for $i=$ $1,2, \ldots, n-k+1$, while $S_{1}, S_{2}, S_{3}$ are defined as follows:

$$
\begin{aligned}
& S_{1}=\sum_{i=1}^{n-k+1} P\left(A_{i}\right), \\
& S_{2}=\sum_{1 \leq i<j \leq n-k+1} P\left(A_{i} A_{j}\right), \\
& S_{3}=\sum_{i<j<v \leq n-k+1} P\left(A_{i} A_{j} A_{\nu}\right) .
\end{aligned}
$$

Theorem 15 (Sfakianakis et al. [18]). Let $R_{r, k, n}^{L}(p)$ denote the reliability function of a linear $r$-within-consecutive- $k$-out-of-n: $F$ system, where $p$ is the common reliability of its components. Then $R_{r, k, n}^{L}(p)$ satisfies the following inequalities:

(i)

$$
R_{r, k, n}^{L}(p) \geq a_{1} S_{1}-a_{2} S_{2}+a_{3} S_{3}
$$

where

$$
\begin{aligned}
a_{1} & =\frac{2(n-k+1)+t-1}{(n-k+1)(t+1)}, \\
a_{2} & =\frac{2(n-k+2 t-1)}{(n-k+1) t(t+1)}, \\
a_{3} & =\frac{6}{(n-k+1) t(t+1)}, \\
t & =\left[\frac{2\left((n-k-1) S_{2}-3 S_{3}\right)}{(n-k) S_{1}-2 S_{2}}\right],
\end{aligned}
$$

(ii)

$$
R_{r, k, n}^{L}(p) \leq \min \left(1, S_{1}-b_{2} S_{2}+b_{3} S_{3}\right),
$$

where

$$
\begin{aligned}
b_{2} & =\frac{2(2 t-1)}{t(t+1)}, \\
b_{3} & =\frac{6}{t(t+1)}, \\
t & =\left[\frac{3 S_{3}}{S_{2}}\right]+2 .
\end{aligned}
$$

For the next Theorem, the following definitions are necessary:

(i) $Z_{i}$ denotes the event that the linear $r$-within-consecutive- $k$-out-of-i: $F$ system consisting of the components $1,2, \ldots, i$ is $\operatorname{good}(i=k, k+1, \ldots, n)$.

(ii) $X_{i}$ denotes the event that the ith component fails and there are at least $r-1$ failures among components $i-$ $k+1, i-k+2, \ldots, i-1,(i=k, k+1, \ldots, n)$. (iii) $B_{i}$ denotes the event that there are at most $r-1$ failures among components $i-k+1, i-k+2, \ldots, i-1,(i=$ $k, k+1, \ldots, n)$.

(iv) $C_{i}$ denotes the event that, for $f=\min (i-k, k-r+1)$, there is no failure among components $(i-k)-f+1,(i-$ $k)-f+2, \ldots, i-k$, for $i=k+1, k+2, \ldots, n$.

Theorem 16 (Papastavridis and Koutras [21]). Let $R_{r, k, n}^{L}(t)$ denote the reliability function of a linear $r$-within-consecutive$k$-out-of- $n: F$ system. Then $R_{r, k, n}^{L}(t)$ satisfies the following inequalities:

$$
\begin{aligned}
& P\left(Z_{k}\right) \prod_{i=k+1}^{n} P\left(X_{i}^{\prime}\right) \\
& \quad \leq R_{r, k, n}^{L}(t) \\
& \quad \leq P\left(Z_{k}\right) \prod_{i=k+1}^{n}\left[1-\gamma_{i} P\left(X_{i}\right)+q_{i} \gamma_{i} P\left(B_{i}^{\prime}\right)\right],
\end{aligned}
$$

where $q_{i}$ is the unreliability of the ith component, while $\gamma_{i}=$ $P\left(C_{i}\right) / P\left(B_{i}\right)$.

2.2.4. Signature Vector of $r$-within-Consecutive- $k$-out-of-n: $F$ Systems. Let $p$ denote the common reliability of the components of an $r$-within-consecutive- $k$-out-of- $n$ : $F$ system. The following theorem offers a generating function approach of the aforementioned system.

Theorem 17. Let $\left(s_{1}(n), s_{2}(n), \ldots, s_{n}(n)\right)$ and $R_{2, k, n}(p)$ be the signature and the reliability function of an 2-withinconsecutive- $k$-out-of- $n$ : $F$ system, respectively. Then

(a) the double generating function of $i\left(\begin{array}{c}n \\ i\end{array}\right) s_{i}(n)$ is given by

$$
\begin{aligned}
& \sum_{n=1}^{\infty} \sum_{i=1}^{n} i\left(\begin{array}{l}
n \\
i
\end{array}\right) s_{i}(n) t^{i} x^{n} \\
& =\frac{t^{2} x\left[2 x-2 x^{2}+((k-1) t-2) x^{k}+(2-k t) x^{k+1}+t x^{2 k}\right]}{(x-1)^{2}\left(1-x-t x^{k}\right)^{2}}
\end{aligned}
$$

(Triantafyllou and Koutras [22]),

(b) the generating function of $R_{2, k, n}(p)$ is given as follows:

$$
R(z ; p)=\frac{1+q z \sum_{j=0}^{k-2}(p z)^{j}}{1-p z-q p^{k-1} z^{k}}
$$

(Koutras [13]).

The next theorem offers expressions for the evaluation of the signature vector of an 2-within-consecutive-k-out-of-n: $F$ system.

Theorem 18 (Triantafyllou and Koutras [22]). Let $\left(s_{1}(n)\right.$, $\left.s_{2}(n), \ldots, s_{n}(n)\right)$ be the signature vector of an 2-withinconsecutive- $k$-out-of-n: $F$ system. Then the following ensues: 
(i) The quantities $q_{i}(n)=(n)_{i} s_{i}(n), i=1,2, \ldots, n$, where $(n)_{i}=n(n-1) \cdots(n-i+1)$, satisfy the recurrence relation:

$$
\begin{aligned}
& q_{i+1}(n+1)=4 q_{i+1}(n)+4 q_{i+1}(n-2)-6 q_{i+1}(n-1) \\
& -q_{i+1}(n-3)+i\left(2\left(q_{i}(n-k+1)\right)-q_{i}(n-k-2)\right. \\
& -3 q_{i}(n-k)+3 q_{i}(n-k-1)-(i-1) \\
& \left.\cdot\left(q_{i}(n-2 k+1)-q_{i}(n-2 k)-q_{i}(n-2 k-1)\right)\right) \\
& \text { for } i=0,1, \ldots, n-1 \text { and } n \geq 2 k+2 .
\end{aligned}
$$

(ii) The quantities $s_{i}(n)$ can be expressed as

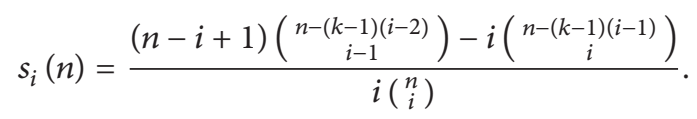

It is worth mentioning that well-performed simulations study of an r-within-consecutive-k-out-of-n: $F$ system has been developed by Eryilmaz et al. [20]. Moreover, Kan et al. [23] studied the circular case of the aforementioned structure and offered a new approximation for its reliability.

2.3. $(n, f, k)$ Systems. An $(n, f, k)$ system involves two common failure criteria. More specifically, it consists of $n$ components (ordered in a line or a circle) and fails if and only if there exist at least $f$ failed components or at least $k$ consecutive failed components. It is worth of mentioning that the configuration of an $(n, f, k)$ system was first introduced by Tung [24] as an application to a complex infrared detecting system and since then it has attracted considerable research attention. In the sequel, we present the main results for $(n, f, k)$ systems appearing in the literature, such as recurrence relations and closed formulas for the evaluation of the reliability function and signature vector. It is worth mentioning that the aforementioned system generalizes the well-known consecutive- $k$-out-of- $n$ : $F$ system (for $f>k$ ), while for $f \leq k$ the $(n, f, k)$ system reduces to an ordinary $f$-out-of- $n$ : $F$ system.

2.3.1. Recursive Schemes for the Reliability of $(n, f, k)$ Systems. The following theorems provide recurrence for the calculation of reliability of an $(n, f, k)$ system.

Theorem 19 (Zuo et al. [25]). Let $A(i, j, k)$ be the event that the $(i, j, k)$ subsystem fails (the subsystem consists of components $1,2, \ldots, i, i \geq j \geq 0, i \geq k)$, while $Q(i, j, k)$ denotes the corresponding failure probability $P(A(i, j, k))$. Then the unreliability function of the $(i, j, k)$ system satisfies the following recurrence relation:

$$
\begin{aligned}
& Q(i, j, k) \\
& =p_{i} Q(i-1, j, k)+q_{i} Q(i-1, j-1, k) \\
& \quad+[1-Q(i-k-1, j-k, k)] p_{i-k} \prod_{l=i-k+1}^{i} q_{l},
\end{aligned}
$$

where $p_{a}\left(q_{a}\right)$ is the reliability (unreliability) of the ath component.

In order to launch the aforementioned recursive scheme, the following set of initial conditions is necessary:

$$
\begin{aligned}
Q(i, j, k) & =0, \quad \text { if } i<\min (j, k), \\
Q(i, j, k) & =1 \quad \text { if } j=0, \\
p_{0} & =1 .
\end{aligned}
$$

The complexity for calculating $Q(n, f, k)$ using the above recurrence relation is equal to $O(n f)$. The next theorem provides an alternative recursive scheme for the evaluation of the reliability function of the $(n, f, k)$ system.

Theorem 20 (Triantafyllou and Koutras [26]). The reliability function $R_{n}$ of an $(n, f, 2)$ system with i.i.d. components satisfies the following recurrence relation:

$$
\begin{aligned}
R_{n} & =\sum_{m=1}^{f}(-p)^{m-1}\left(\begin{array}{c}
f-1 \\
m-1
\end{array}\right) f \frac{m(1-p)+(f+1) p}{m(f-m+1)} \\
& \cdot R_{n-m}+(-p)^{f} R_{n-f-1},
\end{aligned}
$$

where $p$ denotes the common reliability of its components.

In order to launch the recursive scheme established above, an adequate number of initial conditions is necessary. These conditions are given as follows

$$
\begin{aligned}
& R_{n}=1, \quad \text { if } n<\min (f, 2), \\
& R_{n}=0, \quad \text { if } f=0 \text { or } n=0, \\
& R_{n}=R_{f, n: F}, \quad \text { if } f \leq 2,
\end{aligned}
$$

where $R_{f, n: F}$ is the reliability of a $f$-out-of: $n: F$ system; that is, $R_{f, n: F}=\sum_{j=0}^{f-1}\left(\begin{array}{c}n \\ j\end{array}\right) p^{n-j}(1-p)^{j}$.

2.3.2. Exact Formulas for the Reliability of $(n, f, k)$ Systems. Let us first consider an $(n, f, k)$ system, where $f>k$; we recall that for the case $f \leq k$ the $(n, f, k)$ system coincides with the well-known $f$-out-of- $n$ : $F$ system and its reliability properties have been extensively studied in the past. Chang et al. [27] established a Markov chain representation of the $(n, f, k)$ system, which leads to the computation of the reliability function of the aforementioned structure. More specifically, for the $(n, f, k)$ system with $f>k$, let us define the state space for process $\{Y(t), t=0,1, \ldots\}$ as

$$
S=\{(i, j): 0 \leq i \leq k-1, \quad i \leq j \leq f-1\} \cup\left\{s_{N}\right\},
$$

where $(i, j)$ indicates a working state in which the system consisting of components $1,2, \ldots, t$ has $j$ failed components, its last $i-1$ components have failed, and the $(t-i)$ th component is working. State $s_{N}$ indicates the system $(1,2, \ldots, t)$ fails. Then $\{Y(t)\}$ is a Markov chain with transition matrix of the form 


$$
\Lambda_{t}(n)=\left(\begin{array}{ccccc}
A_{f \times f}^{(1)} & B_{f \times(f-1)}^{(1)} & 0 & 0 & C_{f \times 1}^{(1)} \\
A_{(f-1) \times f}^{(2)} & 0 & B_{(f-1) \times(f-2)}^{(2)} & 0 & C_{(f-1) \times 1}^{(2)} \\
\vdots & & & & \\
A_{(f-k+1) \times f}^{(k)} & 0 & 0 & B_{(f-k+1) \times(f-k)}^{(k)} & C_{(f-k+1) \times 1}^{(k)} \\
0 & 0 & 0 & 0 & 1
\end{array}\right)_{N \times N}
$$

where

$$
\begin{aligned}
& A_{(f-i+1) \times f}^{(i)}=\left(\begin{array}{llll}
\underbrace{0 \cdots 0}_{i-1} & p & & \\
& & \ddots & \\
& & & p
\end{array}\right) \text {, } \\
& B_{(f-i+1) \times(f-i)}^{(i)}=\left(\begin{array}{ccc}
p & & \\
& \ddots & \\
& & p \\
& & 0
\end{array}\right) \text {, } \\
& i=1,2, \ldots, k-1 \text {, } \\
& C_{(f-i+1) \times 1}^{(i)}=\left(\begin{array}{lll}
0 \cdots 0 & q
\end{array}\right)^{\prime}, \quad i=1,2, \ldots, k-1
\end{aligned}
$$

and $N=(2 f-k+1) k / 2+1$.

As proved in Koutras [13], the reliability $R_{n}$ of a structure can be expressed as

$$
R_{n}=\pi_{0}^{\prime} \Lambda^{n} \mathbf{u}=1-\pi_{0}^{\prime} \Lambda^{n} \mathbf{e}_{N}
$$

where $\Lambda$ is the transition probability matrix associated to the structure and

$$
\begin{aligned}
\boldsymbol{\pi}_{0} & =(1,0,0, \ldots, 0)^{\prime}, \\
\mathbf{u} & =(1,1, \ldots, 1,0)^{\prime}, \\
\mathbf{e}_{N} & =(0,0, \ldots, 0,1)^{\prime}
\end{aligned}
$$

Therefore, applying the above expression obtained for the transition matrix of an $(n, f, k)$ system, one may easily calculate the reliability function of the aforementioned structure.

Let us next consider the following probabilities:

$$
\begin{aligned}
& \theta_{m}^{(0)}=P\left(X_{1}=\cdots=X_{m}=0\right), \\
& \theta_{m}^{(1)}=P\left(X_{1}=\cdots=X_{m}=1\right),
\end{aligned}
$$

for $m \geq 1$,

where $X_{i}$ is the state of $i$ th component $\left(X_{i} \in\{0,1\}\right)$, for $i=$ $1,2, \ldots, n$. The following theorem offers a closed expression for the evaluation of reliability of an $(n, f, k)$ system.

Theorem 21 (Eryilmaz [28]). The reliability function $R_{n}$ of an $(n, f, k)$ system with exchangeable components can be expressed as follows:

$$
R_{n}=\sum_{i=0}^{f-1} \sum_{j=0}^{n-i} N(i, k, n)(-1)^{j}\left(\begin{array}{c}
n-i \\
j
\end{array}\right) \theta_{i+j}^{(0)},
$$

where

$$
\begin{aligned}
& N(i, k, n) \\
& =\sum_{j=0}^{\min ([i / k], n-i+1)}(-1)^{j}\left(\begin{array}{c}
n-i+1 \\
j
\end{array}\right)\left(\begin{array}{c}
n-j k \\
n-i
\end{array}\right) .
\end{aligned}
$$

In the sequel, we present results for an $(n, f, k)$ system with Markov dependent components. Let $X_{1}, X_{2}, \ldots, X_{n}$ denote the states of the Markov dependent components with transition probabilities

$$
\begin{aligned}
& P\left(X_{i}=0 \mid X_{i-1}=0\right)=p_{00}, \\
& P\left(X_{i}=1 \mid X_{i-1}=0\right)=p_{01}, \\
& P\left(X_{i}=0 \mid X_{i-1}=1\right)=p_{10}, \\
& P\left(X_{i}=1 \mid X_{i-1}=1\right)=p_{11},
\end{aligned}
$$

with $1 \leq i \leq n$ and initial probabilities $p_{1}=P\left(X_{1}=1\right)$, $p_{0}=P\left(X_{1}=0\right)$. If we define $g(n, r, l)$ as

$$
\begin{gathered}
g(n, r, l)=\sum_{t=0}^{1} \sum_{s=0}^{1}\left(\begin{array}{c}
n-l-1 \\
r-t-s
\end{array}\right)\left(p_{00}\right)^{l-r} \\
\cdot p_{01}^{r-s} p_{10}^{r-t}\left(p_{11}\right)^{n-l-r+t+s-1} p_{1-t},
\end{gathered}
$$

the following theorem provides an expression for the evaluation of reliability of $(n, f, k)$ systems.

Theorem 22 (Demir [29]). The reliability function $R_{n}$ of an $(n, f, k)$ system with Markov dependent components can be expressed as follows:

$$
R_{n}=p_{1} p_{11}^{n-1}+\sum_{m=1}^{f-1} \sum_{r=1}^{\min (m, n-m)} N(r, f, m) g(n, r, m) .
$$

2.3.3. Signature Vector of $(n, f, k)$ Systems. Let $p$ denote the common reliability of the components of an $(n, f, k)$ system. The following theorem offers a generating function approach of the aforementioned system. 
Theorem 23 (Triantafyllou and Koutras [26]). Let $\left(s_{1}(n)\right.$, $\left.s_{2}(n), \ldots, s_{n}(n)\right)$ be the signature of an $(n, f, 2)$ system, respectively. Then the double generating function of $i\left(\begin{array}{c}n \\ i\end{array}\right) s_{i}(n)$ is given by

$$
\begin{aligned}
\sum_{n=1}^{\infty} \sum_{i=1}^{n} i\left(\begin{array}{l}
n \\
i
\end{array}\right) s_{i}(n) t^{i} x^{n} & \\
= & \frac{d}{x^{2}(1-x-t x)^{2}\left(1-x-t x^{2}\right)^{2}(1-x)^{f+1}},
\end{aligned}
$$

where

$$
\begin{aligned}
d & =-t^{4}(1-x)^{f+1} x^{6}-f(1-x)^{3}\left(-\left(t x^{2}\right)^{f}+(1\right. \\
& \left.-x)\left(t x^{2}\right)^{f}\right)+t^{3} x^{4}\left(-\left(t x^{2}\right)^{f}(2 x+f-2)+(1\right. \\
& \left.-x)^{f} x\left(x^{2}-1\right)\right)+t(x-1)^{2}\left((1-x)\left(t x^{2}\right)^{f}\right. \\
& \cdot\left((f-1) x^{2}+x+f-2\right)-\left(t x^{2}\right)^{f}\left(2 f x^{2}+2 x\right. \\
& +f-2))+(t x)^{2}\left(-(1-x)^{2}\left(t x^{2}\right)^{f}(x+f-3)\right. \\
& +(x-1)\left(-2(1-x)^{f+1} x^{2}\right. \\
& \left.\left.-\left(t x^{2}\right)^{f}\left(4(x-1)+f\left(x^{2}+2\right)\right)\right)\right) .
\end{aligned}
$$

Theorem 24 offers recursive relations for the evaluation of the signature vector of an $(n, f, 2)$ system.

Theorem 24 (Triantafyllou [30]). The coordinates $s_{i}(n)$ of the signature vector of an $(n, f, 2)$ system satisfy the following recurrence relation:

$$
\begin{aligned}
& \sum_{j=0}^{f+1}\left(\begin{array}{c}
f+1 \\
j
\end{array}\right)(-1)^{j}\left(i\left(\begin{array}{c}
n-j-2 \\
i
\end{array}\right) s_{i}(n-j-2)\right. \\
& -(i-1)\left(\begin{array}{c}
n-j-3 \\
i-1
\end{array}\right) s_{i-1}(n-j-3) \\
& -3 i\left(\begin{array}{c}
n-j-3 \\
i
\end{array}\right) s_{i}(n-j-3) \\
& +3 i\left(\begin{array}{c}
n-j-4 \\
i
\end{array}\right) s_{i}(n-j-4)+2(i-2) \\
& +\left(\begin{array}{c}
n-j-5 \\
i-2
\end{array}\right) s_{i-2}(n-j-5) s_{i-2}(n-j-5) \\
& +3(i-1)\left(\begin{array}{c}
n-j-5 \\
i-1
\end{array}\right) s_{i-1}(n-j-5) \\
& +i\left(\begin{array}{c}
n-j-5 \\
i
\end{array}\right) s_{i}(n-j-5)-(i-2)
\end{aligned}
$$

$$
\begin{aligned}
& \cdot\left(\begin{array}{c}
n-j-6 \\
i-2
\end{array}\right) s_{i-2}(n-j-6)-2(i-1) \\
& \cdot\left(\begin{array}{c}
n-j-6 \\
i-1
\end{array}\right) s_{i-1}(n-j-6)-(i-2) \\
& \cdot\left(\begin{array}{c}
n-j-7 \\
i-2
\end{array}\right) s_{i-2}(n-j-7)-(i-3) \\
& \left.\cdot\left(\begin{array}{c}
n-j-7 \\
i-3
\end{array}\right) s_{i-3}(n-j-7)\right)=0 .
\end{aligned}
$$

Let $T$ be the lifetime of a system whose components' lifetimes are $T_{1}, T_{2}, \ldots, T_{n}$ and $T_{1: n}<T_{2: n}<\cdots<T_{n: n}$ the order statistics associated with them. It is easy to observe that the lifetime of an $(n, f, k)$ system can be represented as a function of the lifetimes of consecutive k-out-of-n: $F$ and $k$-out-of-n: $F$ systems. Generally speaking, the lifetimes of systems involving two common failure criteria can be expressed as either

$$
\begin{aligned}
T_{*} & =\min \left(S, T_{a: n}\right), \\
\text { or } T^{*} & =\max \left(S, T_{a: n}\right),
\end{aligned}
$$

where $S$ denotes the lifetime associated with the system different from a-out-of-n structure but having the same components' lifetimes $T_{1}, T_{2}, \ldots, T_{n}$. The next theorem presents an alternative way of computing the signature of an $(n, f, k)$ system.

Theorem 25 (Eryilmaz and Zuo [31]). Let $\left(s_{1}, s_{2}, \ldots, s_{n}\right)$ be the signature of the system with lifetime $S$. Then the signatures of the systems with lifetimes $T_{*}$ and $T^{*}$ are given, respectively, as

$$
\begin{aligned}
& \mathbf{p}_{*}=\left(s_{1}, s_{2}, \ldots, s_{a-1}, \sum_{i=a}^{n} s_{i}, 0, \ldots, 0\right), \\
& \mathbf{p}^{*}=\left(0, \ldots, 0, \sum_{i=a}^{a} s_{i}, s_{a+1}, \ldots, s_{n}\right) .
\end{aligned}
$$

It is noteworthy that the dual system of an $(n, f, k)$ structure has been studied by Cui et al. [32], while a generalization of these systems, named $(n, f, k)$ systems, with weighted components has been introduced by Eryilmaz and Aksoy [33]. Gera [34] studied reliability systems with two working criteria and presented some results associated with the well-known qualification tests, while Kamalja [35] derived expressions for the Birnbaum importance measures for both structures.

2.4. Reliability Systems with Weighted Components. The idea that all components in a reliability structure are not created equal is seemingly an obvious concept. In other words, it is not quaint to assume that different components may have different failure probabilities. Generally speaking, a reliability system which consists of weighted components, for example, each component carries its own positive weight, fails if and only if the total weight of the failed components exceeds 
a specific benchmark. In the sequel, the literature on weighted reliability structures is briefly reviewed. Consider a system with $n$ components and suppose that the $i$ th component is associated with a weight $w_{i}>0, i=1,2, \ldots, n$. Then the system is still working if and only if the sum of weights of the failed components is less than (or equal to) a certain threshold $T>0$.

2.4.1. Weighted $k$-out-of-n Systems. A weighted $k$-out-of- $n$ : $G(F)$ system consists of $n$ components, each with its own positive weight $w_{i}>0$ (total system weight equal to $w$ ), such that the system works (fails) if and only if the total weight of the working (failed) components is at least $k$. It is noteworthy that the reliability of a weighted $k$-out-of- $n$ : $G$ system is the complement of the unreliability of a weighted $(n-k+1)$ out-of- $n$ : $F$ system. It goes without saying that the $k$-out-of- $n$ : $G(F)$ system is a special case of the corresponding weighted $k$ out-of- $n: G(F)$ system wherein the weight of each component equals 1 . The next theorem offers an efficient algorithm for the evaluation of the reliability of the weighted $k$-out-of- $n$ : $G$ system.

Theorem 26 (Wu and Chen [36]). Let $R(i, j)$ be the reliability of the weighted j-out-of- $i$ : $G$ system, while $w_{i}>0$ is the weight of the ith component. Then if we denote by $p_{i}\left(q_{i}\right)$ the reliability (unreliability) of the ith component, the reliability of the structure satisfies the following recurrence relation:

$$
\begin{aligned}
& R(i, j) \\
& = \begin{cases}p_{i} R\left(i-1, j-w_{i}\right)+q_{i} R(i-1, j), & \text { if } j-w_{i} \geq 0, \\
p_{i}+q_{i} R(i-1, j), & \text { otherwise. }\end{cases}
\end{aligned}
$$

The following theorem provides a similar result concerning the dual structure.

Theorem 27 (Chen and Yang [37]). Let $R(i, j)$ be the reliability of the weighted j-out-of-i: F system, while $w_{i}>0$ is the weight of the ith component. Then if we denote by $p_{i}\left(q_{i}\right)$ the reliability (unreliability) of the ith component, the reliability of the structure satisfies the following recurrence relation:

$$
\begin{aligned}
& R(i, j) \\
& = \begin{cases}0, & \text { if } i \leq 0, j \geq 0, \\
1, & \text { if } i>0, j=0, \\
\left(1-p_{i}\right) R\left(i-w_{j}, j-1\right)+p_{j} R(i, j-1), & \text { otherwise. }\end{cases}
\end{aligned}
$$

An extension of the aforementioned one-stage weighted $k$ out-of- $n$ model has been proposed by Chen and Yang [37]. More specifically, Chen and Yang [37] considered a twostage weighted $k$-out-of-n system, which consists of $m$ subsystems. Each subsystem has a (one-stage) weighted $k$-outof-n structure, which is called the second level structure. The interrelationship between the $m$ subsystems follows a certain coherent structure, which is called the first-level structure. Generally speaking, a two-stage weighted $k$-out-of-n system can be decomposed into two hierarchical levels: the first (higher) level can be of any coherent structure and the second (lower) level has a weighted $k$-out-of- $n$ structure.
An additional generalization of the well-known weighted $k$-out-of- $n$ system has been introduced by Eryilmaz [38]. More specifically, Eryilmaz [38] assumed that the system has a performance level above $c$ if there are at least $k$ working components and the sum of the weights of all working components is above $c$. Among others, Eryilmaz [38] deduced recursive relations for the calculation of the system state probabilities, while a detailed simulation study has been taken into play in order to observe the time spent by the system in state $c$ or above. Finally, Li and Zuo [39] studied the multistate weighted $k$-out-of- $n$ systems, where each component may be in more than 2 states and therefore its contribution to the system's weight can be differentiated analogously.

2.4.2. Consecutive Weighted $k$-out-of-n Systems. A weighted consecutive $k$-out-of- $n$ : $F$ system consists of $n$ components, each with its own positive weight $w_{i}>0$ (total system weight equal to $w$ ), such that the system fails if and only if the total weight of the failed components is at least $k$. It goes without saying that the consecutive $k$-out-of- $n$ : $F$ system is a special case of the corresponding weighted consecutive $k$-out-of- $n$ : $F$ system wherein the weight of each component equals 1 . Efficient algorithms for the evaluation of the reliability of the linear weighted consecutive $k$-out-of- $n$ : $F$ system have appeared in the literature (for more details see Kuo and Zuo [1]), while Samaniego and Shaked [40] extended the idea of weighted components, by giving to the components weights that can take any positive value (not necessary integervalued).

2.5. $\left(\left(n_{1}, n_{2}, \ldots, n_{N}\right), f, k\right)$ Systems. Cui and Xie [41] introduced a generalized $k$-out-of- $n$ system, denoted by $\left(\left(n_{1}, n_{2}, \ldots, n_{N}\right), f, k\right)$. Such a system consists of $N$ modules ordered in a line or a circle, while the $i$ th module is composed of $n_{i} \geq 1$ components in parallel. In other words, the $\left(\left(n_{1}, n_{2}, \ldots, n_{N}\right), f, k\right)$ system fails if and only if there are at least $f$ failed components or at least $k$ consecutive failed modules. It goes without saying that, for $n_{1}=n_{2}=\cdots=n_{N}=1$, an $\left(\left(n_{1}, n_{2}, \ldots, n_{N}\right), f, k\right)$ system reduces to a simple $(N, f, k)$ while for $f=\sum_{i=1}^{N} n_{i}$ coincides with the well-known consecutive $k$-out-of- $N$ : $F$ system and for $N=1$ with the ordinary $f$-out-of- $n$ : $F$ structure. The following theorem offers recursive relations for the evaluation of the reliability function of a linear and circular $\left(\left(n_{1}, n_{2}, \ldots, n_{N}\right), f, k\right)$ system, respectively.

Theorem 28 (Cui and Xie [41]). (i) For a linear $\left(\left(n_{1}, n_{2}, \ldots\right.\right.$, $\left.\left.n_{N}\right), f, k\right)$ system, the reliability function $R_{L}\left(\left(n_{1}, n_{2}, \ldots\right.\right.$, $\left.n_{N}\right), f, k$ ) satisfies the following recurrence:

$$
\begin{gathered}
R_{L}\left(\left(n_{1}, n_{2}, \ldots, n_{N}\right), f, k\right)=\sum_{s=0}^{n_{N}} A(N, s) \\
\cdot R_{L}\left(\left(n_{1}, n_{2}, \ldots, n_{N-1}\right), f-s, k\right)
\end{gathered}
$$




$$
\begin{aligned}
& -\left(\prod_{i=N-k+1}^{N} \prod_{j=1}^{n_{i}}\left(1-p_{i j}\right)\right) \sum_{s=0}^{n_{N-k}-1} A(N-k, s) \\
& \cdot R_{L}\left(\left(n_{1}, n_{2}, \ldots, n_{N-k-1}\right), f-\sum_{i=N-k+1}^{N} n_{i}-s, k\right),
\end{aligned}
$$

where $A(j, s)$ is the probability that, in module $j$, there are $s$ failed components.

(ii) For a circular $\left(\left(n_{1}, n_{2}, \ldots, n_{N}\right), f, k\right)$ system, the reliability function $R_{C}\left(\left(n_{1}, n_{2}, \ldots, n_{N}\right), f, k\right)$ satisfies the following recurrence:

$$
\begin{aligned}
R_{C} & \left(\left(n_{1}, n_{2}, \ldots, n_{N}\right), f, k\right) \\
& =\sum_{n=0}^{s_{N}-1} A(N, s) R_{L}\left(\left(n_{1}, n_{2}, \ldots, n_{N-1}\right), f-s, k\right) \\
& +\prod_{i=1}^{n_{N}}\left(1-p_{N i}\right) R_{C}\left(\left(n_{1}, n_{2}, \ldots, n_{N-1}\right), f-n_{N}, k\right) \\
& -\sum_{i=1}^{k} \sum_{s_{1}=0}^{n_{N-k+l-1}-1} \sum_{s_{2}=0}^{n_{j}-1} A\left(N-k+i-1, s_{1}\right) A\left(i, s_{2}\right) \\
& \times R_{L}\left(n_{i+1}, \ldots, n_{N-k+i-2}\right), f-s_{1}-s_{2} \\
& \left.-\sum_{j=N-k+i}^{N+i-1} n_{j}, k\right)\left(\prod_{l=N-k+i}^{N+i-1} \prod_{j=1}^{n_{i}}\left(1-p_{i j}\right)\right) .
\end{aligned}
$$

It is worth mentioning that the $\left(\left(n_{1}, n_{2}, \ldots, n_{N}\right), f, k\right)$ system can be obtained by adding more components in parallel to the basic components of an $(n, f, k)$ structure. Since the aforementioned system involves multiple failure criteria, Cui and Xie [41] studied the Birnbaum importance with respect to the $k$ th failure criterion as an importance measure computed only under this failure criterion. Firstly, for a reliability system $S$ with $m \geq 1$ failure criteria, they assumed that, for any one of $j(j \leq m)$, the Birnbaum importance is different, while, for any one of $m$ - j failure criteria, the Birnbaum importance is the same. Moreover, Cui and Xie [41] denoted by $A_{1}=\{S$ works under failure criteria $1,2, \ldots, j\}$ and $A_{2}=\{S$ works under failure criteria $j+1, \ldots, m\}$ the specific disjoint events and they proved that the Birnbaum importance of the reliability system $S$ depends upon these j failure criteria. Finally, note that the wellknown series and parallel reliability systems are included in the more general $\left(\left(n_{1}, n_{2}, \ldots, n_{N}\right), f, k\right)$ systems' family as special cases (for $f \geq \max \left\{n_{i}\right\}$ and $k=1$ and $\{N=k$ and $f=$ $\left.\sum_{i=1}^{N} n_{i}\right\}$, resp.).

2.6. $(n, f, k(i, j))$ and $\langle n, f, k(i, j)\rangle$ Systems. Guo et al. [42] generalized the idea of the $(n, f, k)$ system, studied by Chang et al. [27] and Zuo et al. [25]. More specifically, they introduced a reliability structure, named the $(n, f, k(i, j))$ : $F$ system, which consists of $n$ components ordered in a line or circle and fails if and only if there exist at least $f$ failed components or at least $k$ consecutive failed components among components $i, i+1, \ldots, j-1, j$. It is noteworthy that, for the circular case, component index should have modulo $n$ property, that is, components $i$ and $n+i$ indicate the same one, while the following requirements should be satisfied:

$$
\begin{aligned}
& \text { (i) } j-i+1 \geq k \text {, if } j>i \text {, } \\
& \text { (ii) } n+j-i+1 \geq k \text {, if } j<i \text {. }
\end{aligned}
$$

Otherwise, the consecutive $k$ failure criterion can be removed. It goes without saying that when $i=1, j=n$, or $i-j=1$, the $(n, f, k(i, j)): F$ system becomes the well-known $(n, f, k)$ structure. Furthermore, Guo et al. [42] mentioned an additional justification of the aforementioned reliability systems. More precisely, they introduced the $\langle n, f, k(i, j)\rangle: F$ system, which consists of $n$ components ordered in a line or circle, and fails if and only if there exist at least $f$ failed components and at least $k$ consecutive failed components among components $i, i+1, \ldots, j-1, j$. Finally, Guo et al. [42] considered the dual structures of the $(n, f, k(i, j))$ : $F$ and $\langle n, f, k(i, j)\rangle: F$ systems, taking into account the argumentation applied by Cui et al. [32].

The main result of the aforementioned paper is the employment of a two-stage Markov chain in order to give the system reliability in the form of product of matrices. Generally speaking, the finite Markov chain imbedding technique is to embed a Markov chain $\{Y(t)\}$ defined on the state space $S=\{1,2, \ldots, N\}$ and the discrete index space $T=\{1,2, \ldots, n\}$ into a given system, while the system fails if there exists $t_{0}, 1 \leq t_{0} \leq n$, such that $Y(t)=N$, for all $t_{0} \leq t \leq n$.

The unique justification is to divide the discrete index space and the nonabsorbing state space into two disjoined parts. For example, if we consider the $(n, f, k(i, j)): F$ $(\langle n, f, k(i, j)\rangle: F)$ system with component reliabilities $p_{t}\left(q_{t}=\right.$ $\left.1-p_{t}\right), t=1,2, \ldots, n$, by rearranging their components without changing the system reliability, we can transform it into the linear $(n, f, k(n-j+i, n)): F(\langle n, f, k(n-j+i, n)\rangle$ : $F)$ system with component reliabilities $u_{t}\left(v_{t}=1-u_{t}\right)$, where $p_{t}, q_{t}$ and $u_{t}, v_{t}$ satisfy the following relations:

$$
\begin{aligned}
& \left(u_{t}, v_{t}\right) \\
& \quad= \begin{cases}\left(p_{t}, q_{t}\right), & \text { if } 1 \leq t \leq i-1, \\
\left(p_{t-i+j+1}, q_{t-i+j+1}\right), & \text { if } i \leq t \leq n-j+i-1, \\
\left(p_{t-n+j}, q_{t-n+j}\right), & \text { if } n-j+i \leq t \leq n .\end{cases}
\end{aligned}
$$

The rearrangement is as follows by a transform:

$$
\begin{aligned}
& \text { Old-component order }(1,2, \ldots, i-1, i, i \\
& \quad+1, \ldots, j, j+1, j+2, \ldots, n), \\
& \text { New-component order }(1,2, \ldots, i-1, j+1, j \\
& \quad+2, \ldots, n, i, i+1, \ldots, j),
\end{aligned}
$$

where the first line (old-component order) denotes the components order before rearrangement, the second line 
(New-component order) denotes the components order after rearrangement, that is, we move to the end of the line that section to which the consecutive criterion applies so that the new system consists of components $1,2, \ldots, i-1, j+1, j+$ $2, \ldots, n, i, i+1, \ldots, j$ in an ordered line. Thus, the study of the aforementioned reliability structures is covered by the linear case $(n, f, k(n-j+i, n)): F(\langle n, f, k(i, j)\rangle: F)$ system with component reliabilities $u_{t}$.

It is worth noting that Guo et al. [42] presented a detailed study for the evaluation of the reliability function of the $(n, f, k(i, j)): F(\langle n, f, k(i, j)\rangle: F)$ systems, by employing a two-stage Markov chain procedure and illustrating numerical examples.

2.7. Consecutive k-out-of-n: $F$ Systems with Cycle $k$. An $r$ consecutive $k$-out-of- $n$ : $F$ system consists of $n$ linearly arranged components and the system fails if and only if at least $r$ nonoverlapping runs of $k$ components fail. Boland and Papastavridis [43] studied the case where there are $k$ distinct components with failure probabilities $q_{i}, i=1,2, \ldots, k$ and where the failure probability of the $j$ th component is $q_{i}(j=$ $m k+i(1 \leq i \leq k))$. In other words, they focused on the special case of an $r$ consecutive $k$-out-of- $n$ : $F$ system where there is a "cyclical" pattern in failure probabilities of the components. More precisely, Boland and Papastavridis [43] were interested in how the order of $q_{1}, q_{2}, \ldots, q_{k}$ affects the failure probability of this new structure, named $r$ consecutive $k$-out-of- $n$ : $F$ system with cycle $k$.

Let us next denote by $F(n, r)$ the failure probability of an $r$ consecutive $k$-out-of- $n$ : $F$ system with cycle $k$, while $p_{i}\left(q_{i}\right)$ is the reliability (unreliability) of the $i$ th component. The following theorems offer recursive expressions for the unreliability of the aforementioned structure.

Theorem 29 (Boland and Papastavridis [43]). Consider an $r$ consecutive $k$-out-of- $n$ : $F$ system with cycle $k$, where $n=m k+i$, $1 \leq i \leq k$ and $m \geq 0$. Then for any integer $r$ (except for $r=m+1$ and $i=k$ ), the unreliability function of the system satisfies the following recurrence:

$$
\begin{aligned}
& F(m k+i, r) \\
& \quad=F(m k+i-1, r) \\
& \quad+\sum_{t=0}^{1} \sum_{s=1}^{m}(-1)^{t} p_{i} Q^{s} F((m-s) k+i-1, r-s+t),
\end{aligned}
$$

where $Q=\prod_{i=1}^{k} q_{i}$.

Theorem 30 (Boland and Papastavridis [43]). Consider an $r$ consecutive $k$-out-of-n: $F$ system with cycle $k$, where $1 \leq i \leq k$ and $m \geq 0$. Then the unreliability function of the system satisfies the following recurrence:

$$
\begin{aligned}
& F(m k+i, r)=F(m k, r) \\
& \quad+\sum_{j>0} \sum_{t \in(-\infty, \infty)} \sum_{s_{1}, s_{2}, \ldots, s_{j}}(-1)^{t}\left(\begin{array}{l}
j \\
t
\end{array}\right) \sigma_{j}(i) Q^{s_{1}+s_{2}+\cdots+s_{j}}
\end{aligned}
$$

$$
\begin{aligned}
& \times F\left(\left(m-s_{1}-s_{2}-\cdots-s_{j}\right) k, r-s_{1}-\cdots-s_{j}\right. \\
& +t) .
\end{aligned}
$$

It is clear from the above result that the unreliability $F(n, r)$ is independent of the order $q_{1}, q_{2}, \ldots, q_{k}$ when $n$ is a multiple of k. For $n=m k+1$, it follows that

$$
\begin{aligned}
F(m k+1, r)= & F(m k, r) \\
& +p_{1} \sum_{t=0}^{1} \sum_{s} Q^{s} F((m-s) k, r-s+t)
\end{aligned}
$$

and therefore we deduce that the quantity $F(n, r)=F(m k+1, r)$ is maximized by maximizing $p_{1}$ or, generally speaking, the $F(n, r)$ is maximized (minimized) when $q_{1} \leq q_{2} \leq \cdots \leq q_{k}$ $\left(q_{1} \geq q_{2} \geq \cdots \geq q_{k}\right)$. It is worth mentioning that Boland and Papastavridis [43] provided additionally interesting applications of the $r$ consecutive $k$-out-of- $n$ : $F$ system with cycle $k$ for the arrangement of sport competitions and inspections procedures in quality control.

2.8. $m$-Consecutive $k$-out-of- $n$ : $F$ Systems with Overlapping Runs. Agarwal and Mohan [44] introduced and studied the $m$-consecutive- $k$-out-of- $n$ : $F$ system with overlapping runs. This reliability structure consists of $n$ linearly ordered components and fails if and only if there exist at least $m$ overlapping sequences of $k$ consecutive failed components ( $n \geq m+k-1)$. It is straightforward that for $m=$ 1 the aforementioned system coincides with an ordinary consecutive- $k$-out-of- $n$ : $F$ structure. Graphical Evaluation and Review Technique (GERT) analysis is used for reliability evaluation of the system for both i.i.d. and $(k-1)$-step Markov dependent components, in a unified manner. More specifically, Agarwal and Mohan [44] proved the following results:

(i) For a $m$-consecutive- $k$-out-of- $n$ : $F$ system with overlapping runs composed by i.i.d components, the generating function of the waiting time for the occurrence of the system failure is given as

$$
\begin{aligned}
& W_{0, m+k-1}(0, z)=\sum_{j=0}^{m-1}\left(\begin{array}{c}
m-1 \\
j
\end{array}\right) \\
& \cdot \frac{q^{m+k+j(k-1)-1} p^{j} z^{m+k+k j-1}}{\left(1-p z-q p z^{2}-q^{2} p z^{3}-\cdots-q^{k-1} p z^{k}\right)^{j+1}} .
\end{aligned}
$$

Hence the reliability function of the above structure can be expressed as

$$
R_{m_{0}}(n)=1-\sum_{u=m+k-1}^{n} \xi(u),
$$

where $\xi(u)$ is the coefficient of $z^{u}$ in the power series expansion of the generating function $W_{0, m+k-1}(0, z)$. 
(ii) For a $m$-consecutive- $k$-out-of- $n$ : $F$ system with overlapping runs composed by $(k-1)$-step Markov dependent components, the generating function of the waiting time for the occurrence of the system failure is given as

$$
\begin{aligned}
& W_{0, m+k-1}^{k-1}(0, z)=\sum_{j=0}^{m-1}\left(\begin{array}{c}
m-1 \\
j
\end{array}\right) \\
& \cdot \frac{\left(q_{0} q_{1} \cdots q_{k-2} z^{k-1}\right)^{j+1}\left(q_{k-1} z\right)^{m} p_{k-1}^{j} z^{j}}{\left(1-p_{0} z-q_{0} p_{1} z^{2}-\cdots-q_{0} q_{1} \cdots q_{k-2} p_{k-1} z^{k}\right)^{j+1}} .
\end{aligned}
$$

Hence, the reliability function of the above structure can be expressed as

$$
R_{m_{0}}^{k-1}(n)=1-\sum_{u=m+k-1}^{n} \xi(u)
$$

where $\xi(u)$ is the coefficient of $z^{u}$ in the power series expansion of the generating function $W_{0, m+k-1}^{k-1}(0, z)$.

In addition, Eryilmaz [45] derived an explicit combinatorial expression for the number of its path sets including a specified number of working components. More specifically, Eryilmaz [45] proved the following result.

Theorem 31 (Eryilmaz [45]). The number of path sets $r_{i}(n)$ of an $m$-consecutive-k-out-of-n: $F$ system with overlapping runs including $i$ working components can be expressed as follows:

$$
\begin{aligned}
& r_{i}(n)=N(i+1, k+1, n+1) \\
& +\sum_{x=1}^{m-1} \sum_{a=1}^{m i n(i+1, x)}\left(\begin{array}{c}
i+1 \\
a
\end{array}\right)\left(\begin{array}{c}
x-1 \\
a-1
\end{array}\right) \\
& \cdot N(i-a+1, k+1, n-x-a k+1),
\end{aligned}
$$

where $n-z_{\phi} \leq i<n, r_{n}(n)=1\left(z_{\phi}=n-1-[(n-m-k+1) / k]\right)$ and

$$
\begin{aligned}
& N(a, b, c) \\
& =\sum_{j=0}^{\min (a,[(c-a) /(b-1)])}(-1)^{j}\left(\begin{array}{l}
a \\
j
\end{array}\right)\left(\begin{array}{c}
c-j(b-1)-1 \\
a-1
\end{array}\right) .
\end{aligned}
$$

Having at hand the above expression for the determination of $r_{i}(n)$, one may easily compute important characteristics of the $m$-consecutive- $k$-out-of-n: $F$ system with overlapping runs, such as its reliability function or its signature vector. Indeed, let us first denote by $z_{\phi}$ the maximum number of failed components such that the system with structure function $\phi$ can still work successfully and by $p$ the common reliability of its components. Since the reliability and the signature of a coherent structure can be expressed as

$$
\begin{gathered}
R=\sum_{i=n-z_{\phi}}^{n} r_{i}(n) p^{i}(1-p)^{n-i}, \\
s_{i}(n)=\frac{r_{n-i+1}(n)}{\left(\begin{array}{c}
n \\
n-i+1
\end{array}\right)}-\frac{r_{n-i}(n)}{\left(\begin{array}{c}
n \\
n-i
\end{array}\right)},
\end{gathered}
$$

respectively (for more details, see Boland [46] and Samaniego [3]), it is goes without saying that Theorem 31 leads immediately to the desired results.

2.9. Combined $m$-Consecutive $k$-out-of- $n$ : $F$ and Consecutive$k_{c}$-out-of-n: F Systems. Mohan et al. [47] proposed and studied a new model, named the combined $m$-consecutive$k$-out-of- $n$ : $F$ and consecutive- $k_{c}$-out-of- $n$ : $F$ systems. This reliability structure consists of $n$ linearly ordered components and fails if and only if there exist at least $k_{c}$ consecutive failed components or at least $m$ nonoverlapping runs of $k$ consecutive failed components, where $k_{c}<m k$. By assuming that the components are i.i.d., Mohan et al. [47] employed the well-known GERT analysis to evaluate the reliability function and presented interesting applications of the aforementioned structure in several areas, such as signal processing or bank automatic payment systems. More specifically, the failure probability of the aforementioned system is explained as a tree structure, while the direction of the calculating procedure is from the root node to a leaf node via intermediate ones. The main steps of the algorithm can be briefly described as follows:

(i) At step 1, the failure probability at root node $x_{0}$ is determined as

$$
F\left(x_{0}\right)_{0}=\sum_{u=k_{c}}^{n} \xi(u)
$$

where $\xi(u)$ is the coefficient of $z^{u}$ in the power series expansion of the generating function of the waiting time for the occurrence of the system failure at root node $x_{0}$.

(ii) At step 2, the failure probability at node ${ }^{j}\left(x_{0}\right)_{t}$ is deduced as follows:

$$
F^{j}\left(x_{0}\right)_{t}=\sum_{u=k_{c}+k \sum_{s=1}^{i} i_{s j}+t}^{n} \xi(u)
$$

where $\xi(u)$ is the coefficient of $z^{u}$ in the power series expansion of the generating function of the waiting time for the occurrence of the system failure at root $\operatorname{node}^{j}\left(x_{0}\right)_{t}$.

(iii) At step 3, the total failure probability of the system is calculated as

$$
F\left(n, k_{c}, m k\right)=F\left(x_{0}\right)_{0}+\sum_{\text {all nodes generated }} F^{j}\left(x_{i}\right)_{t} \text {. }
$$

It is noteworthy that the complexity of the proposed algorithm for the calculation of the reliability of a combined $m$-consecutive- $k$-out-of- $n: F$ and consecutive- $k_{c}$-out-of- $n$ : $F$ systems has been determined to be equal to $O\left(\left[\left(n-k_{c}\right) /(k+\right.\right.$ $\left.1)]^{3}\right)$. For an up-to-date overview of GERT analysis results of consecutive- $k$ systems, the interested reader is referred to Sen et al. [48].

Moreover, Eryilmaz [49] derived an explicit expression for the number of its path sets including a specified number 
of working components. More specifically, Eryilmaz [49] proved the following result.

Theorem 32 (Eryilmaz [49]). The number of path sets $r_{i}(n)$ of a combined m-consecutive- $k$-out-of- $n$ : $F$ and consecutive- $k_{c^{-}}$ out-of-n: $F$ systems with i.i.d. components including $i$ working ones can be expressed as follows:

$$
\begin{aligned}
r_{i}(n) & \\
= & \sum_{x=0}^{m-1} \sum_{s=1}^{\min (i+1,[(n+1) / 2])}\left(\begin{array}{c}
i+1 \\
s
\end{array}\right) C\left(s, k, k_{c}, n-i, x\right),
\end{aligned}
$$

where $z_{\min } \leq i<n, r_{n}(n)=1\left(z_{\min }\right.$ indicates the minimum number of failed components at the moment of system failure) and the quantities $C\left(s, k, k_{c}, l, x\right)$ can be calculated via the following recurrence:

$$
\begin{aligned}
& C\left(s, k, k_{c}, l, x\right) \\
& =\sum_{a=1}^{k-1} C\left(s-1, k, k_{c}, l-a, x\right) \\
& \quad+\sum_{a=k}^{k_{c}-1} C\left(s-1, k, k_{c}, l-a, x-\left[\frac{a}{k}\right]\right),
\end{aligned}
$$

with

$$
\begin{aligned}
C & \left(1, k, k_{c}, l, x\right) \\
& = \begin{cases}1, & \text { if } 0<l<k, x=0, \\
1, & \text { if } k x \leq l<\min \left(k_{c}, k x+k\right), x>0, \\
0, & \text { otherwise. }\end{cases}
\end{aligned}
$$

Having at hand the above expression for $r_{i}(n)$ and applying the well-known equalities for the evaluation of reliability function and signature of a coherent system in terms of $r_{i}(n)$ (these equations have been already mentioned in previous section), one may easily compute these important characteristics of the combined $m$-consecutive- $k$-out-of-n: $F$ and consecutive- $k_{c}$ out-of-n: F systems with i.i.d. components. Finally, note that Eryilmaz [49] studied also the aforementioned structure under the assumption of Markovian type dependence between its components and derived an expression for its reliability.

2.10. Constrained $(k, d)$-out-of-n Systems. Eryilmaz and Zuo [50] proposed and studied two new models, which generalize the well-known $k$-out-of- $n$ : $F$ and consecutive $k$-out-of- $n$ : $G$ systems. These extensions consider an additional constraint on the number of working components between successive failures. More precisely, in addition to the working conditions of $k$-out-of- $n$ : $F$ and consecutive $k$-out-of- $n$ : $G$ systems there must be at least $d$ consecutive working components between any of two successive failures.

The first reliability structure introduced by Eryilmaz and Zuo [50] is called a constrained $(k, d)$-out-of- $n$ : $F$ system consisting of $n$ linearly ordered components. This system fails if and only if there at least $k$ failed components or there are less than $d$ consecutive working components between any of two failed ones. It goes without saying that, for $d=0$, the constrained $(k, d)$-out-of- $n$ : $F$ system reduces to the ordinary $k$-out-of- $n$ : $F$ system.

Let $\Omega_{n}^{[d, \infty)}$ denote the set of all binary sequences of length $n$ where the runs of successes between two consecutive failures have length at least $d$. Then the reliability of constrained $(k, d)$-out-of- $n$ : $F$ system is given as follows:

$$
R_{k, d, n}^{F}=P\left\{\mathbf{X} \in \Omega_{n}^{[d, \infty)}, S_{n}^{(0)}<k\right\},
$$

where $\mathbf{X}=\left(X_{1}, X_{2}, \ldots, X_{n}\right)$ denotes the states of components $\left(X_{i}=1\right.$ if the $i$ th component is operating, and $X_{i}=0$, otherwise), while $S_{n}^{(0)}$ represents the total number of failed components. The following theorem offers expressions for the evaluation of the reliability function of the constrained $(k, d)$-out-of- $n$ : $F$ system with i.i.d. and Markov dependent components, respectively.

Theorem 33 (Eryilmaz and Zuo [50]). (i) For a constrained $(k, d)$-out-of- $n$ : $F$ system consisting of $n$ independent and identical components with common reliability $p$, the reliability function is given as follows:

$$
\quad R_{k, d, n}^{F}
$$

(ii) For a constrained $(k, d)$-out-of-n: $F$ system consisting of $n$ Markov dependent components with transition probabilities $p_{r s}=P\left(X_{i}=s \mid X_{i-1}=r\right), r, s \in\{0,1\}, i=2,3, \ldots, n$, and initial probabilities $p_{0}=P\left(X_{1}=0\right), p_{1}=P\left(X_{1}=1\right)$, the reliability function is given as follows:

$$
\begin{aligned}
R_{k, d, n}^{F}= & \sum_{i=0}^{1} \sum_{j=0}^{1} \sum_{l=i+j}^{k-1}\left(\begin{array}{c}
n-d(l-1)-2 \\
l-i-j
\end{array}\right) \\
\cdot & p_{11}^{n-2 l+i+j-1} p_{10}^{l-i} p_{01}^{l-j}\left(1-p_{i}\right) .
\end{aligned}
$$

Moreover, an alternative reliability structure introduced by Eryilmaz and Zuo [50] is called a constrained consecutive $(k, d)$-out-of- $n$ : $G$ system consisting of $n$ linearly ordered components. This system works if and only if there at least $k$ consecutive working components and there are a least $d$ consecutive working components between any of two failed ones. It goes without saying that, for $d=0$, the constrained consecutive $(k, d)$-out-of- $n$ : $G$ system reduces to the ordinary consecutive $k$-out-of- $n$ : $G$ system.

Let $L_{(n)}^{1}$ denote the longest run of successes. Then the reliability of a constrained consecutive $(k, d)$-out-of- $n$ : $G$ system is given as follows:

$$
R_{k, d, n}^{G}=P\left\{\mathbf{X} \in \Omega_{n}^{[d, \infty)}, L_{n}^{(1)} \geq k\right\},
$$

where $\mathbf{X}=\left(X_{1}, X_{2}, \ldots, X_{n}\right)$ denotes the states of components. Theorem 34 offers expressions for the evaluation of the reliability function of the constrained consecutive $(k, d)$-outof- $n$ : $G$ system with i.i.d. and Markov dependent components, respectively. 
Theorem 34 (Eryilmaz and Zuo [50]). (i) For a constrained consecutive $(k, d)$-out-of-n: $G$ system consisting of $n$ independent and identical components with common reliability $p$, the reliability function is given as follows:

$$
\begin{aligned}
& R_{k, d, n}^{G}=\sum_{l=0}^{[(n+d) /(d+1)]}\left(\begin{array}{c}
n-d(l-1) \\
l
\end{array}\right) p^{n-l}(1-p)^{l} \\
& \quad-\sum_{l=0}^{n} A_{l+1, n-l}(k-1, k-d-1, \ldots, k-d-1, k \\
& \quad-1) p^{n-l}(1-p)^{l},
\end{aligned}
$$

where

$$
\begin{aligned}
A_{n, k}\left(u_{1}, u_{2}, \ldots, u_{n}\right) & \\
= & \left(\begin{array}{c}
n+k-s-1 \\
n-1
\end{array}\right) \\
& \quad+\sum_{r=1}^{n}(-1)^{r} \sum\left(\begin{array}{c}
n+k-s-u_{i 1}-u_{i 2}-r-1 \\
n-1
\end{array}\right) .
\end{aligned}
$$

(ii) For a constrained consecutive $(k, d)$-out-of-n: $G$ system consisting of $n$ Markov dependent components with transition probabilities

$$
\begin{aligned}
& p_{r s}=P\left(X_{i}=s \mid X_{i-1}=r\right), \\
& r, s \in\{0,1\}, i=2,3, \ldots, n
\end{aligned}
$$

and initial probabilities $p_{0}=P\left(X_{1}=0\right), p_{1}=P\left(X_{1}=1\right)$, the reliability is given as

$$
\begin{aligned}
& R_{k, d, n}^{G}=R_{n+1, d, n}^{F}-\sum_{l} A_{l+1, n-l}(k-2, k-d-1, \ldots, k \\
& -d-1, k-2) p_{1} p_{11}^{n-2 l-1} p_{10}^{l} p_{01}^{l}-\sum_{l} A_{l, n-l}(k-2, k \\
& \quad-d-1, \ldots, k-d-1) p_{1} p_{11}^{n-2 l} p_{10}^{l} p_{01}^{l-1} \\
& \quad-\sum_{l} A_{l, n-l}(k-d-1, \ldots, k-d-1, k-2) p_{0} \\
& \cdot p_{11}^{n-2 l} p_{10}^{l-1} p_{01}^{l}-\sum_{l} A_{l-1, n-l}(k-d-1, \ldots, k-d \\
& \text { - 1) } p_{0} p_{11}^{n-2 l+1} p_{10}^{l-1} p_{01}^{l-1} .
\end{aligned}
$$

It is noteworthy that Eryilmaz and Zuo [50] proved explicit formulas for the calculation of the signature of both constrained $(k, d)$-out-of-n systems they considered.

2.11. Combined $m_{f_{1}}$-Consecutive $k_{c f_{1}}$-out-of- $n$ and $m_{f_{2}}$-Consecutive- $k_{c f_{2}}$-out-of- $n$ Systems. The common $m$-consecutive $k_{c}$-out-of- $n$ : $F$ system consists of $n$ linearly ordered components and fails if and only if there exist at least $m$ nonoverlapping runs of $k_{c}$ consecutive failed components. Gera [51] considered a more general reliability model, named the combined $m_{f_{1}}$-consecutive $k_{c f_{1}}$-out-of- $n$ and $m_{f_{2}}$ consecutive- $k_{c f_{2}}$-out-of- $n$ systems. The new structure consists of $n$ components and fails if and only if there exist at least $m_{f_{1}}$ nonoverlapping runs of $k_{c f_{1}}$ consecutive failed components or at least $m_{f_{2}}$ nonoverlapping runs of $k_{c f_{2}}$ consecutive failed ones. Gera [51] shed light on the cases where $m_{f_{1}} \geq m_{f_{2}}, k_{c f_{1}} \leq k_{c f_{2}}$, and additionally $k_{c f_{1}}$ is not an integer divisor of $k_{c f_{2}}$; that is, $k_{c f_{2}} / k_{c f_{1}}$ is not integer-valued. It goes without saying that otherwise the aforementioned system reduces to an ordinary $m$-consecutive $k_{c}$-out-of- $n$ structure.

Let $Z$ denote the total number of components such that the system fails due to the specific requirements as mentioned, while $X_{z}$ represents the state of the $z$ th component $\left(X_{z}=1(0)\right.$ for success (failure)). In order to determine the reliability function of combined $m_{f_{1}}$-consecutive $k_{c f_{1}}$-out-of$n$ and $m_{f_{2}}$-consecutive- $k_{c f_{2}}$-out-of- $n$ systems, it is necessary to evaluate the following probabilities:

$$
\begin{aligned}
& P_{0}\left(m_{f_{1}}, m_{f_{2}}, z\right) \\
& \quad=P\left(\text { exactly } m_{f_{1}} \text { times strings of length } k_{c f_{1}},\right. \\
& \left.\quad \text { exactly } m_{f_{2}} \text { times strings of length } k_{c f_{2}}, X_{z}=0\right), \\
& P_{1}\left(m_{f_{1}}, m_{f_{2}}, z\right) \\
& \quad=P\left(\text { exactly } m_{f_{1}} \text { times strings of length } k_{c f_{1}},\right. \\
& \left.\quad \text { exactly } m_{f_{2}} \text { times strings of length } k_{c f_{2}}, X_{z}=1\right) .
\end{aligned}
$$

Gera [51] managed to express the above quantities as follows:

$$
\begin{aligned}
& P_{0}\left(m_{f_{1}}, m_{f_{2}}, z\right)=\sum_{a=1}^{a_{\max }} \sum_{r=0}^{m_{f_{1}}} \sum_{t=0}^{m_{f_{2}}}\left\{q^{a} \delta\left[\left[\frac{a}{k_{c f_{1}}}\right]-r\right] \cdot \delta\left[\left[\frac{a}{k_{c f_{2}}}\right]-t\right] P_{1}\left(m_{f_{1}}-r, m_{f_{2}}-t, z-a\right)\right\} \\
& \quad+q^{z}\left\{u\left[z-m_{x}\right]-u\left[z-m_{n}\right]\right\} \\
& P_{1}\left(m_{f_{1}}, m_{f_{2}}, z\right)=\sum_{b=1}^{s-1} p^{b} P_{0}\left(m_{f_{1}}, m_{f_{2}}, z-b\right),
\end{aligned}
$$


where $u[\cdot], \delta[\cdot]$ are the unit step and delta function, respectively, and $p(q)$ is the common reliability (unreliability) of the components, while

$$
a_{\max }= \begin{cases}z-1, & m_{x} \leq z<m_{n} \\ m_{n}-1, & m_{n} \leq z\end{cases}
$$

The following theorem gives the reliability of any subsystem including $z$ elements of a combined $m_{f_{1}}$-consecutive $k_{c f_{1}}$-outof- $n$ and $m_{f_{2}}$-consecutive- $k_{c f_{2}}$-out-of- $n$ systems.

Theorem 35 (Gera [51]). For combined $m_{f_{1}}$-consecutive $k_{c f_{1}}$ out-of- $n$ and $m_{f_{2}}$-consecutive- $k_{c f_{2}}$-out-of- $n$ systems consisting of $n$ independent and identical components with common reliability $p$, the reliability function of any subsystem including $z$ elements is given as follows:

$$
P(Z>z)=\sum_{i=0}^{m_{f_{1}}-1} \sum_{j=0}^{m_{f_{2}}-1}\left(P_{0}(i, j, z)+P_{1}(i, j, z)\right) .
$$

2.12. Linear $m$-Consecutive $k$-out-of-r-from-n: $F$ Systems. Levitin and Dai [52] proposed a new reliability model that generalizes the linear $r$-within-consecutive- $k$-out-of- $n$ : $F$ system (or consecutive- $k$-out-of- $r$-from- $n$ : $F$ system) to the case of $m$ consecutive overlapping runs of $r$ elements. More specifically, the linear $m$-consecutive $k$-out-of- $r$-from$n$ : $F$ system consists of $n$ linearly ordered $s$-independent components and fails if and only if in each one of at least $m$ consecutive overlapping groups of $r$ consecutive elements at least $k$ of them fail.

For the evaluation of the reliability function $R$ of the aforementioned structure, Levitin and Dai [52] noticed that the following equality ensues

$$
\begin{aligned}
R & =P\left\{\sum_{h=1}^{n-r-m+2}\left[\prod_{s=h}^{h+m-1} 1\left(\sum_{j=s}^{s+r-1}\left(1-X_{j}\right) \geq k\right)\right]\right. \\
& =0\}
\end{aligned}
$$

and developed an efficient algorithm for calculating the function $R$, by employing a procedure based on the universal $z$ transform (for more details about the universal moment generating function approach, the interested reader is referred to Ushakov [53]). It is noticeable that the linear $m$-consecutive $k$-out-of- $r$-from- $n$ : $F$ system meets interesting applications in different areas, such as heating systems' modeling.

2.13. Linear $m$-Gap-Consecutive $k$-out-of-r-from-n: $F$ Systems. Levitin [54] introduced a new reliability model that generalizes the linear $r$-within-consecutive- $k$-out-of- $n$ : $F$ system (or consecutive- $k$-out-of- $r$-from- $n$ : $F$ system). More specifically, the linear $m$-gap-consecutive $k$-out-of- $r$-from$n$ : $F$ system consists of $n$ linearly ordered, identical, and statistically independent components and fails if and only if the gap between any pair of groups of $r$ consecutive elements containing at least $k$ failed components is less than $m$ components. For the evaluation of the reliability function of the aforementioned structure, Levitin [54] employed the universal moment generating function technique and deduced a recursive algorithm that leads to the exact value of system's reliability.

2.14. Linear m-Consecutive-k, l-out-of-n: F Systems. Eryilmaz and Mahmoud [55] proposed a new reliability model that generalizes the linear $m$-consecutive- $k$-out-of- $n$ : $F$ system to the case of $l$-overlapping runs. More specifically, the linear $m$-consecutive $k$, $l$-out-of- $n$ : $F$ system consists of $n$ linearly ordered components and fails if and only if there are at least $m l$-overlapping runs of $k$ consecutive failed components $(n \geq$ $m(k-l)+l, l<k)$. The number of $l$-overlapping runs of length $k$, each of which may have a part of length at most $l$ overlapping with the previous run of length $k$. The inclusion of the new system parameter $l$ provides flexibility for wider application of the model.

Moreover, Eryilmaz and Mahmoud [55] derived an explicit expression for the number of its path sets including a specified number of working components. More specifically, they proved the following result.

Theorem 36 (Eryilmaz and Mahmoud [55]). The number of path sets $r_{i}(n)$ of a linear $m$-consecutive $k$, l-out-of-n: $F$ system including $i$ working components can be expressed as follows:

$$
\begin{aligned}
& r_{i}(n)=C(n-i ; i+1,0 ; k-1 ; k-1) \\
& +\sum_{s=1}^{m-1} \sum_{a=1}^{\min (i+1)}\left(\begin{array}{c}
i+1 \\
a
\end{array}\right)\left(\begin{array}{c}
n-1 \\
a-1
\end{array}\right) \times C(n-i-a l \\
& \quad-(k-l) s ; a, i-a+1 ; k-l-1 ; k-1),
\end{aligned}
$$

for $n-z_{\phi} \leq i \leq n$, where $z_{\phi}$ denotes the maximum number of failed components such that the system can still work successfully, and the quantities $C\left(\beta ; a, r-a ; m_{1}-1, m_{2}-1\right)$ can be calculated via the following formula (see, e.g., Makri et al. [56]):

$$
\begin{aligned}
C & \left(\beta ; a, r-a ; m_{1}-1, m_{2}-1\right) \\
= & \sum_{j_{1}=0}^{\left[a / m_{1}\right]} \sum_{j_{2}=0}^{\left[\left(\beta-m_{1} j_{1}\right) / m_{2}\right]}(-1)^{j_{1}+j_{2}}\left(\begin{array}{c}
a \\
j_{1}
\end{array}\right)\left(\begin{array}{c}
r-a \\
j_{2}
\end{array}\right) \\
& \cdot\left(\begin{array}{c}
\beta-m_{1} j_{1}-m_{2} j_{2}+r-1 \\
r-1
\end{array}\right) .
\end{aligned}
$$

Having at hand the above expression for $r_{i}(n)$ and applying the well-known equalities for the evaluation of reliability and signature of a coherent system in terms of $r_{i}(n)$ (see, e.g., Triantafyllou and Koutras [57]), one may easily compute these important characteristics of the linear $m$-consecutive $k$, l-outof- $n$ : F system. 
2.15. Sparsely Connected Consecutive-k Systems. Zhao et al. [58] introduced generalized consecutive- $k$ systems with sparse $d$. The consecutive failures with sparse $d$ are defined as follows. When components are ordered in a line, two adjacent failed components have no failed components between them and the number of working components between the two failed components is equal to $d$; then the two failed components are called consecutive failures with sparse $d$. Zhao et al. [58] proposed the following reliability models: (i) the linear consecutive $k$-out-of- $n$ : $F$ system with sparse $d$, which consists of $n$ linearly ordered components and fails if and only if there exist at least $k$ consecutive failures with sparse $d$. For the aforementioned structure, Zhao et al. [58] employed the wellknown Markov Chain Imbedding technique in order to achieve expressions for the reliability function of such systems. Moreover, Mohan et al. [59] proved that the generating function of the waiting time for the occurrence of the system failure is given as

$$
W_{0, m+k-1}(0, z)=\frac{(q z)^{k}\left(1-(p z)^{d+1}\right)^{k-1}\left(1-z+q z(p z)^{d+1}\right)}{(1-p z)\left((1-z)(1-p z)^{k-1}+(q z)^{k}(p z)^{d+1}\left(1-(p z)^{d+1}\right)^{k-1}\right)} .
$$

Hence, the reliability function of the above structure can be expressed as

$$
R(n, k)_{d}=1-\sum_{u=k}^{n} \xi(u),
$$

where $\xi(u)$ is the coefficient of $z^{u}$ in the power series expansion of the generating function $W_{0, k}^{d}(0, z)$.

(ii) The $m$-consecutive $k$-out-of- $n$ : $F$ system with sparse $d$ consists of $n$ linearly ordered components and fails if and only if there exist at least $m$ nonoverlapping runs of $k$ consecutive failures with sparse $d$. For the aforementioned structure, Zhao et al. [58] employed the well-known Markov Chain Imbedding technique in order to achieve expressions for the reliability function of such systems. Moreover, Mohan et al. [59] proved that the generating function of the waiting time for the occurrence of the system failure is given as

$$
W_{0, k^{m}}^{d}(0, z)=\left(W_{0, k}^{d}(0, z)\right)^{m} .
$$

(iii) The linear $(n, f, k): F$ system with sparse $d$ consists of $n$ linearly ordered components and fails if and only if there exist at least $f$ total failures or at least $k$ consecutive failures with sparse $d$. For the aforementioned structure, Zhao et al. [58] employed the well-known Markov Chain Imbedding technique in order to achieve expressions for the reliability function of such systems. Moreover, Mohan et al. [59] proposed an algorithm for the evaluation of the corresponding reliability function.

It is worth mentioning that, in case of $d=0$, the consecutive$k$ systems with sparse $d$ reduce to the ordinary consecutive $k$-out-of-n: $F$ structures.

\section{Applications}

Applications of reliability models can range from electrical or engineering and mechanical field up to several contexts referring to humans' activity. For example, an emergency backup power supply in a hospital or a power production at a specific voltage at the microscale may be viewed as direct implementations of reliability modeling. Moreover, doubleloop computer networks, such as the daisy chain or the brained ring, can be studied through the lines of a reliability structure.

Let us consider a television screen characterized by a certain number of pixels and assume that the visual quality is not acceptable if and only if one of the following ensues:

(i) there exist at least $f$ failed pixels,

(ii) there exist at least $k$ consecutive failed pixels.

It is clear that the aforementioned device can be studied as an $(n, f, k)$ system.

In addition, it is of some interest that vacuum systems in accelerators or belt conveyors in open-cast mining operate within the framework of the well-known consecutive $k$ out-of- $n$ system. Among applications of $k$-out-of- $n$ systems, the design of electronic circuits such as very large scale integrated (VLSI) and the automatic repairs of faults in an online system would be the most conspicuous. This type of structure consists of several parallel outputs channeled through a decision-making device that provides the required system function as long as at least a predetermined number $k$ of $n$ parallel outputs are in agreement.

It is worth mentioning that Kalyan and Kumar [60] studied in detail the redundancy optimization in consecutive $k$-out-of- $n$ : $F$ systems. More specifically, for a consecutive $k$-out-of- $n$ structure that has a fluctuation of demand on the system performance, Kalyan and Kumar [60] considered extra resources in order to improve the system performance and developed alternative methods for the maximization of the structures' reliability. It goes without saying that the reliability systems studied in the article of Kalyan and Kumar [60] can be classed under the so-called Protean systems.

Zhang et al. [61] presented an application of the wellknown consecutive $k$-out-of- $n$ : $G$ system to a railroad operation. More specifically, they studied a railroad system consisting of 17 lines (numbered from 1 to 17), which was formulated 


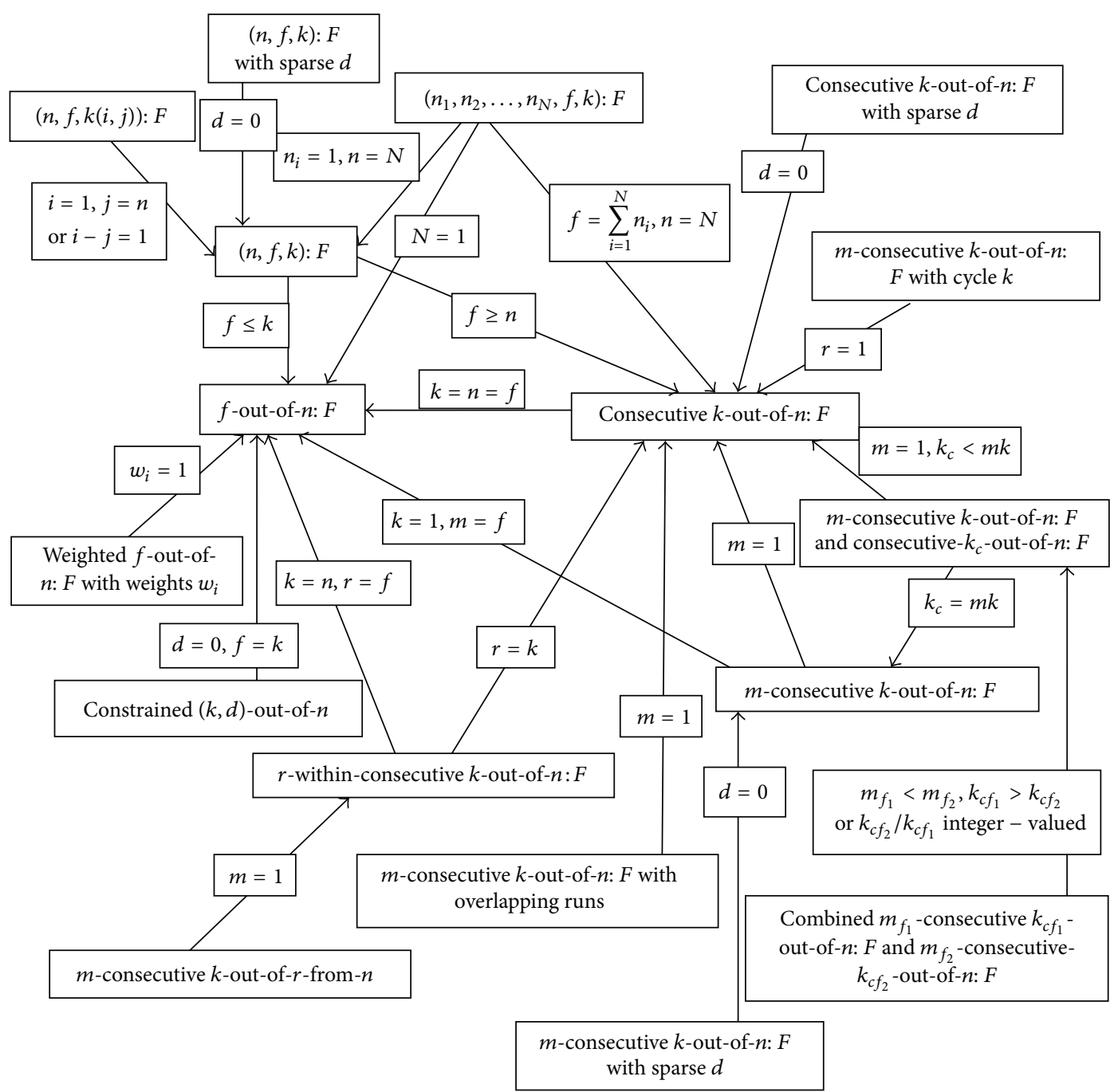

FIGURE 1: Connection between members of the consecutive-type systems' family.

as a reliability study of a linear consecutive $k$-out-of- $n$ : $G$ structure.

Zuo et al. [62] reported an application of $k$-out-of- $n$ : $F$ and consecutive- $k$-out-of- $n$ : $F$ systems in evaluation of the lifetime distribution of furnaces used in a petrochemical company. More precisely, different scenarios were investigated in modeling the reliability behaviors of the furnaces as a function of the reliabilities of the tubes used in the furnaces.

Lu and Liu [63] used the well-known $k$-out-of- $n$ : $G$ structure to quantify the reliability benefit of redundancy units in main circuit of prevailing static generators, called Static Synchronous Compensators (STATCOMs). The fundamental aim of applying a reliability study in the aforementioned operation is to maintain specific parameters of the electric power system by using the variation of the outputs of the so-called STATCOM and describe the effect of the redundancy technique to the total device reliability performance. More specifically, Lu and Liu [63] provided the probabilistic model of the 50 MVA STATCOM devices which were brought into operation at a certain substation in Shanghai, China.
Many safety-critical applications including nuclear power plants are equipped with $k$-out-of- $n$ or specific-voting-logic redundant safety signal generation systems for ensuring both safety and economy. Kang and Kim [64] developed a quantification method for the evaluation of the unreliability of a reactor protection system (RPS), while several configurations of $k$-out-of- $n$ models are investigated.

\section{How Members of the Consecutive-Type Systems' Family Are Connected}

In the literature, there exist a lot of references that mark down the connection between two well-known reliability systems. It is really often for a newly introduced reliability structure to coincide with an older one under some specific restrictions concerning their design parameters. In this section, we attempt to harvest all information that refers to relations among all members of the generalized consecutivetype systems' family.

Figure 1 displays for each system that is included in the consecutive-type systems' family the way that is connected 
to one or more of its members. It goes without saying that both the $k$-out-of- $n$ system and the consecutive $k$-out-of- $n$ system play a crucial role in the aforementioned group of reliability structures. For example, based on Figure 1, one may easily observe that the constrained $(k, d)$-out-of- $n$ system reduces to the well-known $k$-out-of- $n$ structure if its design parameters are adjusted properly (e.g., $d=0, f=k$ ).

\section{Conflict of Interests}

The author declares that there is no conflict of interests regarding the publication of this paper.

\section{Acknowledgments}

The author is grateful to the Editor X. Li and the referees for their helpful comments and suggestions, which led to some improvements of the paper.

\section{References}

[1] W. Kuo and M. J. Zuo, Optimal Reliability Modeling: Principles and Applications, John Wiley \& Sons, New York, NY, USA, 2003.

[2] J. G. Chang, L. Cui, and F. K. Hwang, Reliabilities of Consecutive$k$ Systems, Kluwer Academic Publishers, Dordrecht, The Netherlands, 2000.

[3] F. J. Samaniego, "On closure of the IFR class under formation of coherent systems," IEEE Transactions on Reliability, vol. R-34, no. 1, pp. 69-72, 1985.

[4] J. Navarro and T. Rychlik, "Reliability and expectation bounds for coherent systems with exchangeable components," Journal of Multivariate Analysis, vol. 98, no. 1, pp. 102-113, 2007.

[5] S. Kochar, H. Mukerjee, and F. J. Samaniego, "The signature of a coherent system and its application to comparisons among systems," Naval Research Logistics, vol. 46, no. 5, pp. 507-523, 1999.

[6] S. Eryilmaz and I. Bayramoglu, "On extreme residual lives after the failure of the system," Mathematical Problems in Engineering, vol. 2012, Article ID 342940, 11 pages, 2012.

[7] W. S. Griffith, "On consecutive- $k$-out-of- $n$ : failure systems and their generalizations," in Reliability and Quality Control, A. P. Basu, Ed., pp. 157-165, Elsevier, Amsterdam, The Netherlands, 1986.

[8] S. Papastavridis, “ $m$-consecutive- $k$-out-of- $n: F$ systems," IEEE Transactions on Reliability, vol. 39, no. 3, pp. 386-388, 1990.

[9] P. D. Alevizos, S. Papastavridis, and P. Sypsas, "Reliability of cyclic $m$-consecutive- $k$-out-of- $n: F$ system," in Proceedings of the IASTED International Conference on Reliability, Quality Control and Risk Assessment, H. Pham and M. H. Hamza, Eds., pp. 140143, Washington, DC, USA, November 1992.

[10] F. S. Makri and A. N. Philippou, "Exact reliability formulas for linear and circular $m$-consecutive- $k$-out-of- $n: F$ systems," Microelectronics Reliability, vol. 36, no. 5, pp. 657-660, 1996.

[11] A. P. Godbole, "Approximate reliabilities of $m$-consecutive- $k$ out-of-n: failure systems," Statistica Sinica, vol. 3, no. 2, pp. 321327, 1993.

[12] S. Eryilmaz, M. V. Koutras, and I. S. Triantafyllou, "Signature based analysis of $m$-consecutive- $k$-out-of- $n$ : $F$ systems with exchangeable components," Naval Research Logistics, vol. 58, no. 4, pp. 344-354, 2011.
[13] M. V. Koutras, "On a Markov chain approach for the study of reliability structures," Journal of Applied Probability, vol. 33, no. 2, pp. 357-367, 1996.

[14] S. Eryilmaz, "Component importance for linear consecutive- $k$ out-of- $n$ and $m$-consecutive- $k$-out-of- $n$ systems with exchangeable components," Naval Research Logistics, vol. 60, no. 4, pp. 313-320, 2013.

[15] N. Ghoraf, "On reliability of circular ' $r$-consecutive- $k$-out-of$n$ : F systems," International Journal of Reliability, Quality and Safety Engineering, vol. 20, no. 4, Article ID 1350014, 2013.

[16] I. Greenberg, "The first occurrence of $n$ successes in $N$ trials," Technometrics, vol. 12, no. 3, pp. 627-634, 1970.

[17] B. Saperstein, "On the occurrences of successes within $N$ Bernoulli trials," Technometrics, vol. 15, no. 4, pp. 809-818, 1973.

[18] M. Sfakianakis, S. Kounias, and A. Hillaris, "Reliability of a consecutive $k$-out-of- $r$-from- $n: F$ system," IEEE Transactions on Reliability, vol. 41, no. 3, pp. 442-447, 1992.

[19] S. Eryilmaz, "Consecutive $k$-within- $m$-out-of- $n$ :F system with nonidentical components," Mathematical Problems in Engineering, vol. 2012, Article ID 106359, 8 pages, 2012.

[20] S. Eryilmaz, C. Kan, and F. Akici, "Consecutive $k$-within- $m$-outof- $n: F$ system with exchangeable components," Naval Research Logistics, vol. 56, no. 6, pp. 503-510, 2009.

[21] S. G. Papastavridis and M. V. Koutras, "Bounds for reliability of consecutive $k$-within- $m$-out-of- $n$ : $F$ systems," IEEE Transactions on Reliability, vol. 42, no. 1, pp. 156-160, 1993.

[22] I. S. Triantafyllou and M. V. Koutras, "Signature and IFR preservation of 2-within-consecutive $k$-out-of- $n: F$ systems," IEEE Transactions on Reliability, vol. 60, no. 1, pp. 315-322, 2011.

[23] C. Kan, C. Franko, and C. M. Ozkut, "Reliability and some characteristics of circular consecutive- $k$-within- $m$-out-of- $n: \mathrm{F}$ system," Communications in Statistics: Simulation and Computation, vol. 43, no. 5, pp. 1078-1093, 2014.

[24] S. S. Tung, "Combinatorial analysis in determining reliability," in Proceedings of the Annual Reliability And Maintainability Symposium, pp. 262-266, New York, NY, USA, 1982.

[25] M. J. Zuo, D. Lin, and Y. Wu, "Reliability evaluation of combined $k$-out-of- $n: F$, consecutive- $k$-out-of- $n: F$ and linear connected$(r, s)$-out-of- $(m, n): F$ system structures," IEEE Transactions on Reliability, vol. 49, no. 1, pp. 99-104, 2000.

[26] I. S. Triantafyllou and M. V. Koutras, "Reliability properties of $(n, f, k)$ systems," IEEE Transactions on Reliability, vol. 63, no. 1, pp. 357-366, 2014.

[27] G. J. Chang, L. Cui, and F. K. Hwang, "Reliabilities for $(n, f, k)$ systems," Statistics \& Probability Letters, vol. 43, no. 3, pp. 237242, 1999.

[28] S. Eryilmaz, "Lifetime of combined $k$-out-of- $n$, and consecutive $k_{c}$-out-of- $n$ systems," IEEE Transactions on Reliability, vol. 57, no. 2, pp. 331-335, 2008.

[29] S. Demir, "Reliability of combined $k$-out-of- $n$ and consecutive$k_{c}$-out-of- $n: F$ systems of Markov dependent components," IEEE Transactions on Reliability, vol. 58, no. 4, pp. 691-693, 2009.

[30] I. S. Triantafyllou, "Reliability study of military operations: methods and application," in Military Logistics: Research Advances and Future Trends, V. Zeimpekis, G. Kaimakamis, and N. J. Daras, Eds., vol. 56, pp. 159-170, Springer, Berlin, Germany, 2015.

[31] S. Eryilmaz and M. J. Zuo, "Computing and applying the signature of a system with two common failure criteria," IEEE Transactions on Reliability, vol. 59, no. 3, pp. 576-580, 2010. 
[32] L. Cui, W. Kuo, J. Li, and M. Xie, "On the dual reliability systems of $(n, f, k)$ and $\langle n, f, k\rangle$, , Statistics \& Probability Letters, vol. 76, pp. 1081-1088, 2006.

[33] S. Eryilmaz and T. Aksoy, "Reliability of linear $(n, f, k)$ systems with weighted components," Journal of Systems Science and Systems Engineering, vol. 19, no. 3, pp. 277-284, 2010.

[34] A. E. Gera, "Combined $k$-out-of- $n: \mathrm{G}$, and consecutive $k_{c}$-outof- $n$ :G systems," IEEE Transactions on Reliability, vol. 53, no. 4, pp. 523-531, 2004.

[35] K. K. Kamalja, "Birnbaum reliability importance for and system," Communications in Statistics: Theory \& Methods, vol. 43, pp. 2406-2418, 2014.

[36] J.-S. Wu and R.-J. Chen, "Algorithm for computing the reliability of weighted- $k$-out-of- $n$ systems," IEEE Transactions on Reliability, vol. 43, no. 2, pp. 327-328, 1994.

[37] Y. Chen and Q. Yang, "Reliability of two-stage weighted- $k$-outof- $n$ systems with components in common," IEEE Transactions on Reliability, vol. 54, no. 3, pp. 431-440, 2005.

[38] S. Eryilmaz, "On reliability analysis of a $k$-out-of- $n$ system with components having random weights," Reliability Engineering and System Safety, vol. 109, pp. 41-44, 2013.

[39] W. Li and M. J. Zuo, "Reliability evaluation of multi-state weighted k-out-of- $n$ systems," Reliability Engineering and System Safety, vol. 93, no. 1, pp. 160-167, 2008.

[40] F. J. Samaniego and M. Shaked, "Systems with weighted components," Statistics \& Probability Letters, vol. 78, no. 6, pp. 815-823, 2008.

[41] L. Cui and M. Xie, "On a generalized $k$-out-of- $n$ system and its reliability," International Journal of Systems Science, vol. 36, no. 5, pp. 267-274, 2005.

[42] Y. Guo, L. Cui, J. Li, and S. Gao, "Reliabilities for and systems," Communication in Statistics: Theory \& Methods, vol. 35, pp. 1779-1789, 2006.

[43] P. J. Boland and S. Papastavridis, "Consecutive $k$-out-of- $n: F$ systems with cycle $k$," Statistics \& Probability Letters, vol. 44, no. 2, pp. 155-160, 1999.

[44] M. Agarwal and P. Mohan, "GERT analysis of $m$-consecutive- $k$ out-of- $n: F$ system with overlapping runs and ( $k-1)$-step Markov dependence," International Journal of Operational Research, vol. 3, no. 1-2, pp. 36-51, 2008.

[45] S. Eryilmaz, " $m$-consecutive- $k$-out-of- $n: F$ system with overlapping runs: signature-based reliability analysis," International Journal of Operational Research, vol. 15, no. 1, pp. 64-73, 2012.

[46] P. J. Boland, "Signatures of indirect majority systems," Journal of Applied Probability, vol. 38, no. 2, pp. 597-603, 2001.

[47] P. Mohan, M. Agarwal, and K. Sen, "Combined $m$-consecutive$k$-out-of- $n$ : $F$ and consecutive- $k_{c}$-out-of- $n$ : $F$ Systems," IEEE Transactions on Reliability, vol. 58, pp. 328-337, 2009.

[48] K. Sen, M. Agarwal, and P. Mohan, "GERT analysis of consecutive- $k$ systems: an overview," in Statistical Paradigms: Recent advances and Reconciliations, A. S. Gupta, T. Samanta, and A. Basu, Eds., pp. 293-280, World Scientific, Singapore, 2015.

[49] S. Eryilmaz, "Reliability of combined $m$-consecutive- $k$-out-of$n: F$ and consecutive $k_{c}$-out-of- $n$ systems," IEEE Transactions on Reliability, vol. 61, pp. 215-219, 2012.

[50] S. Eryilmaz and M. J. Zuo, "Constrained $(k, d)$-out-of- $n$ systems," International Journal of Systems Science, vol. 41, no. 6, pp. 679-685, 2010.

[51] A. E. Gera, "Combined $m_{1}$-consecutive- $k_{c 1}$-out-of- $n$ and $m_{2}$ consecutive- $k_{c 2}$-out-of- $n$ systems," IEEE Transactions on Reliability, vol. 60, no. 2, pp. 493-497, 2011.
[52] G. Levitin and Y. Dai, "Linear $m$-consecutive $k$-out-of- $r$-fromn:F systems," IEEE Transactions on Reliability, vol. 60, no. 3, pp. 640-646, 2011.

[53] I. Ushakov, "Universal generating function," Soviet Journal of Computer and Systems Sciences, vol. 24, pp. 118-129, 1986.

[54] G. Levitin, "Linear $m$-gap-consecutive $k$-out-of- $r$-from- $n$ :F systems," Reliability Engineering and System Safety, vol. 96, no. 2, pp. 292-298, 2011.

[55] S. Eryilmaz and B. Mahmoud, "Linear $m$-consecutive- $k$, $l$-outof- $n: F$ system," IEEE Transactions on Reliability, vol. 61, no. 3, pp. 787-791, 2012.

[56] F. S. Makri, A. N. Philippou, and Z. M. Psillakis, "Polya, inverse Polya, and circular Polya distributions of order $k$ for $l$ overlapping success runs," Communications in Statistics: Theory \& Methods, vol. 36, no. 4, pp. 657-668, 2007.

[57] I. S. Triantafyllou and M. V. Koutras, "On the signature of coherent systems and applications," Probability in the Engineering and Informational Sciences, vol. 22, no. 1, pp. 19-35, 2008.

[58] X. Zhao, L. Cui, and W. Kuo, "Reliability for sparsely connected consecutive- $k$ systems," IEEE Transactions on Reliability, vol. 56, no. 3, pp. 516-524, 2007.

[59] P. Mohan, M. Agarwal, and K. Sen, "Reliability analysis of sparsely connected consecutive- $k$ systems: GERT approach," in Proceedings of the 8th International Conference on Reliability, Maintainability and Safety (ICRMS '09), R. Kang, H. Huang, M. J. Zuo, and Q. Mioo, Eds., pp. 213-218, IEEE, Chengdu, China, July 2009.

[60] R. Kalyan and S. Kumar, "A study of protean systemsredundancy optimization in consecutive- $k$-out-of- $n: F$ systems," Microelectronics Reliability, vol. 30, pp. 635-638, 1990.

[61] W. Zhang, C. Miller, and W. Kuo, "Application and analysis for a consecutive- $k$-out-of- $n: G$ structure," Reliability Engineering and System Safety, vol. 33, no. 2, pp. 189-197, 1991.

[62] M. Zuo, S. Chiovelli, and J. Huang, "Reliability evaluation of furnace systems," Reliability Engineering and System Safety, vol. 65, no. 3, pp. 283-287, 1999.

[63] Z. Lu and W. Liu, "Reliability evaluation of STATCOM based on the $k$-out-of- $n$ : $G$ model," in Proceedings of the International Conference on Power System Technology (PowerCon '06), pp. 1-6, IEEE, Chongqing, China, October 2006.

[64] H. G. Kang and H. E. Kim, "Unavailability and spurious operation probability of $k$-out-of- $n$ reactor protection systems in consideration of CCF," Annals of Nuclear Energy, vol. 49, pp. 102-108, 2012. 

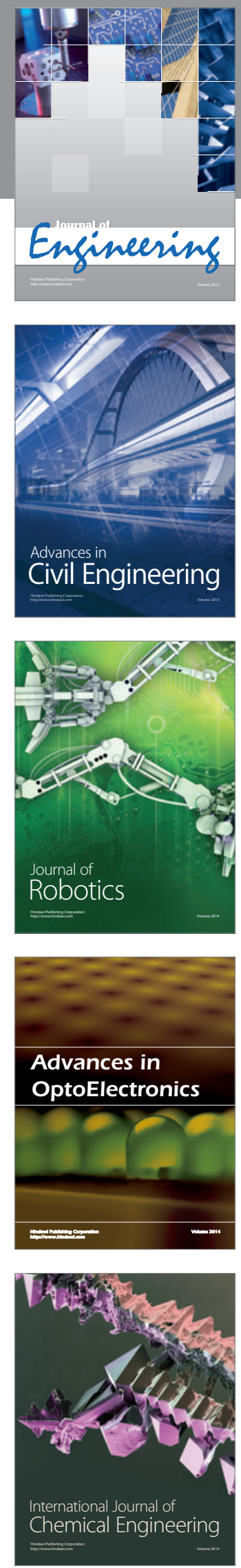

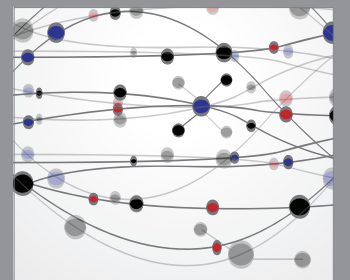

The Scientific World Journal
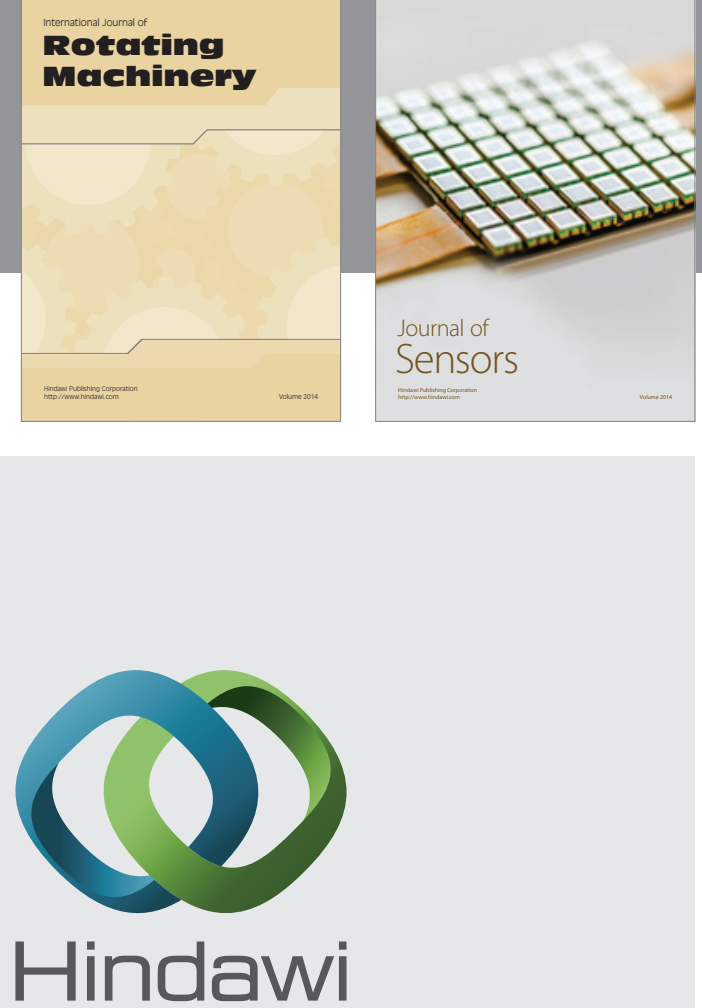

Submit your manuscripts at http://www.hindawi.com
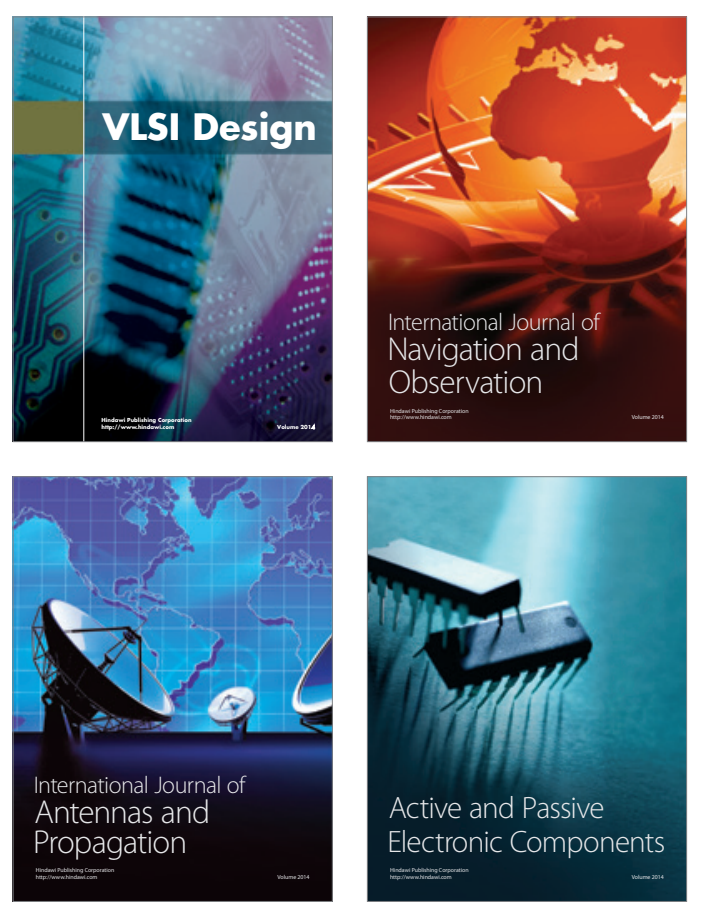
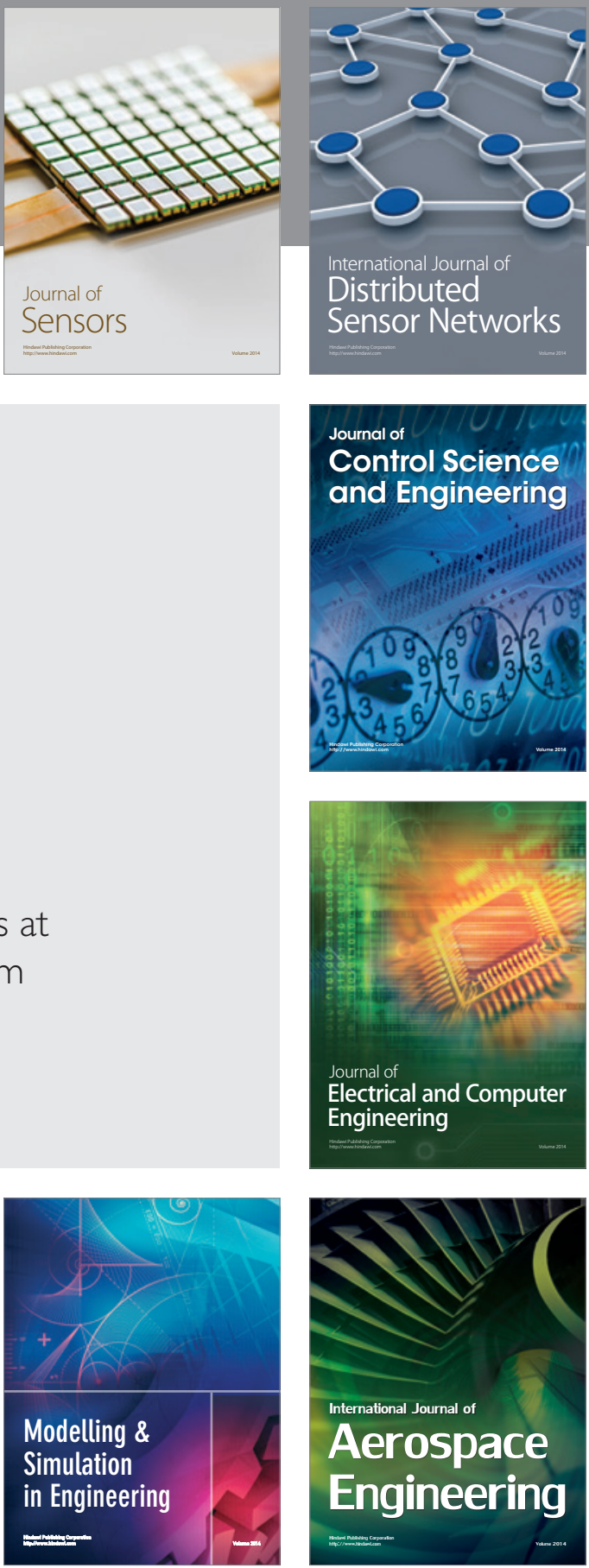

Journal of

Control Science

and Engineering
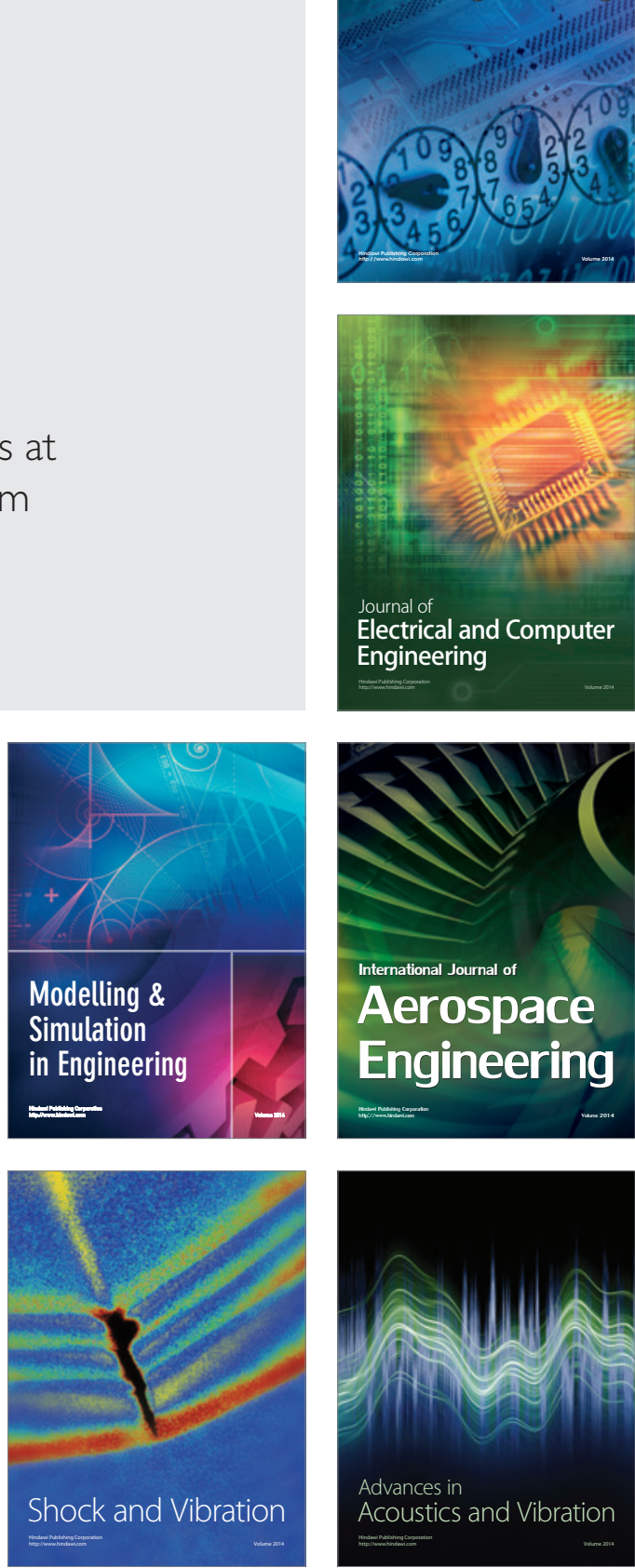